\title{
Study on Spatial-temporal Variation of Atmospheric Pollution in China between
}

\section{4 and 2019}

\author{
Guo Peng ${ }^{(1)}$ Umarova Aminat Batalbievna ${ }^{(2)}$ Luan Yunqi ${ }^{(3)}$
}

(1) (2) (3)Moscow State University of Lomonosov, Department of Soil Physics, Moscow, 119991)

Abstract: The study in this paper reveals that the atmospheric contaminants in mainland China is of concentricity in spatial distribution, persistence in temporal distribution and correlation between different parameters. This spatial-temporal variation law plays an important role in improving and addressing the problem with atmospheric environment of given cities and regions by employing focused and pointed measures. In this paper, seven kinds of atmospheric pollution parameters including PM2.5, PM10, AQI, CO, NO2, O3 and SO2 in 370 Chinese cities from 2014 to 2019 are analyzed based on their hourly mass concentration. The spatial-temporal variations of each parameter in each separated year are obtained by using interpolation calculation towards the annual atmospheric pollution parameters. The results show that higher mass concentration (including the highest mass concentration) of PM2.5,AQI, PM10, CO, NO2, SO2 mainly concentrated in Beijing-Tianjin-Hebei region in the northeast of mainland China and its neighboring regions and Xinjiang region in the northwest of mainland China. The spatial variation of PM2.5, AQI, PM10, CO, NO2, SO2 experienced similar trend. Higher mass concentration (including the highest mass concentration) of O3 mainly concentrated in Qinghai and Inner Mongolia in the central 
north of mainland China and Shandong on the right side of Beijing-Tianjin-Hebei region. The spatial variation of $\mathrm{O} 3$ experienced different trend from that of other parameters. PM2.5, AQI, PM10 indicated increase and decrease, followed by increase and decrease again with time, which was a S-shaped change. Almost the same temporal variation happened to $\mathrm{PM} 2.5$, $\mathrm{AQI}, \mathrm{PM} 10$ and to $\mathrm{CO}, \mathrm{NO} 2, \mathrm{SO}$, which was opposite to O3. The analysis from the perspective of the annual highest mass concentration of PM2.5, AQI, PM10, NO2 and $\mathrm{O} 3$ indicates the atmospheric environment of mainland China had not been authentically improved by 2019. The analysis from the perspective of the annual highest mass concentration of $\mathrm{CO}$ and $\mathrm{SO} 2$ indicates the atmospheric environment of mainland China had been authentically improved by 2019. What should we do immediately is to strengthen environmental governance and address the source of contamination in Beijing-Tianjin-Hebei region in the northeast of China, Xinjiang region in the northwest of China, Qinghai and Inner Mongolia regions in the central north of China.

Keywords: atmospheric particulates, contaminated gas, spatial-temporal variation, Beijing-Tianjin-Hebei region, Xinjiang region

Introduction: At present, a great number of cities in China confront severe situation associated with air pollution, which has become an environmental and social problem drawing much concerns of the general public ${ }^{[1-2]}$. In general, the relative humidity of 
fog is higher than $90 \%$, but the haze would come into being with this index less than $80 \%$ and atmospheric particulates increasing. In people's daily life, the concentration of atmospheric particulates could witness an upward trend due to many industrial, agricultural and architectural practices, such as the emission of contaminants from power plants, the combustion of such biomass as straw ${ }^{[19]}$, the development of real state and the construction of various infrastructures. Among these, PM2.5, a chief culprit, is a kind of so fine atmospheric particulate (aerodynamic diameter is no more than $2.5 \mu \mathrm{m}$ ) that it could easily pass through the nasal cavity and respiratory tract and directly enter bronchi and pulmonary alveoli. Also, it is of great difficulty for our bodies to release them by depending on metabolic process ${ }^{[6]}$. Meanwhile, the large superficial area and strong adsorption capacity of PM2.5 make itself become a mighty carrier to diffuse microorganisms like bacteria and virus ${ }^{[19]}$, organic cyanides, dioxins ${ }^{[3]}$, heavy metals ${ }^{[3,14,17]}$ and other kinds of carcinogenic substances. Long-term exposure to such substances could increase the risk of getting cancer to a large extent ${ }^{[4]}$. Also, regional pollution ${ }^{[8.9]}$ induced by $\mathrm{CO}, \mathrm{NO} 2, \mathrm{SO} 2, \mathrm{O} 3^{[10.11]}$ is increasingly remarkable. In some pivotal cities located in the Beijing-Tianjin-Hebei region and its surrounding areas, Xinjiang, Qinghai, Inner Mongolia, developed Yangtze River Delta region and Pearl River Delta region, haze pollution is frequent and continuous and leads to visibility reduction in a long period ${ }^{[12-13]}$. The air quality of these cities is not only far worse than that of developed countries in Europe and America, but also worse than that of other regions in China. 


\section{Sample collection and analysis approach}

According to the data derived from the Ministry of Ecology and Environment of the People's Republic of China ${ }^{[24]}$, Beijing Municipal Environmental Monitoring Center $^{[23]}$, Beijing Bureau of Statistics ${ }^{[21]}$, the hourly atmospheric pollution parameters related to $\mathrm{PM} 2.5, \mathrm{PM} 10, \mathrm{AQI}, \mathrm{CO}, \mathrm{NO} 2, \mathrm{O} 3, \mathrm{SO} 2$ in 370 Chinese cities from the year 2014 to 2019 were collected in this paper. The analysis approach employed here is to implement Radial Basis Functions (RBF)-based interpolation calculation towards the annual atmospheric pollution parameters of each category through Arcgis. As a consequence, atmospheric pollution parameters in mainland China could be shown in the form of spatial-temporal distribution diagrams. By further statistics, the top ten cities with higher mass concentration of each air pollution parameter per year between 2014 and 2019 were selected out of the 370 cities, and the frequency of their occurrences was also evaluated and analyzed.

\section{Discussion and analysis}

\subsection{The spatial-temporal variation of PM2.5 in mainland China from 2014 to 2019}

From Figure 1, it can be seen that the Beijing-Tianjin-Hebei region in the northeast of mainland China and Xinjiang region in the northwest of mainland China showed higher mass concentration (including the highest mass concentration) of pm2.5 

from the year 2014 to 2019 . There was a similar trend between the spatial variation of PM2.5 and it of AQI, PM10, $\mathrm{CO}, \mathrm{NO} 2, \mathrm{SO} 2$ analyzed in the following content, but that of O3 would indicate a different trend compared with PM2.5. The highest annual mass concentration of PM2.5 was $161 \mu \mathrm{g} \cdot \mathrm{m}-3,201 \mu \mathrm{g} \cdot \mathrm{m}-3,204 \mu \mathrm{g} \cdot \mathrm{m}-3,187 \mu \mathrm{g} \cdot \mathrm{m}-$ 3, $217 \mu \mathrm{g} \cdot \mathrm{m}-3,183 \mu \mathrm{g} \cdot \mathrm{m}-3$ respectively in every separated year between 2014 and 2019. The highest mass concentration of PM2.5 in the year 2018 and 2014 were the highest and lowest among the six years, reaching $217 \mu \mathrm{g} \cdot \mathrm{m}-3$ and $161 \mu \mathrm{g} \cdot \mathrm{m}-3$ respectively. In addition, this index represented increase and then decrease, followed by an increase and decrease again, a kind of S-shaped change with time. The temporal variation of PM2.5 had a similar trend to that of AQI, PM10. On the other hand, from the perspective of the annual highest mass concentration of PM2.5, it can be analyzed that the atmospheric condition of mainland China had not been authentically improved until the year 2019 .
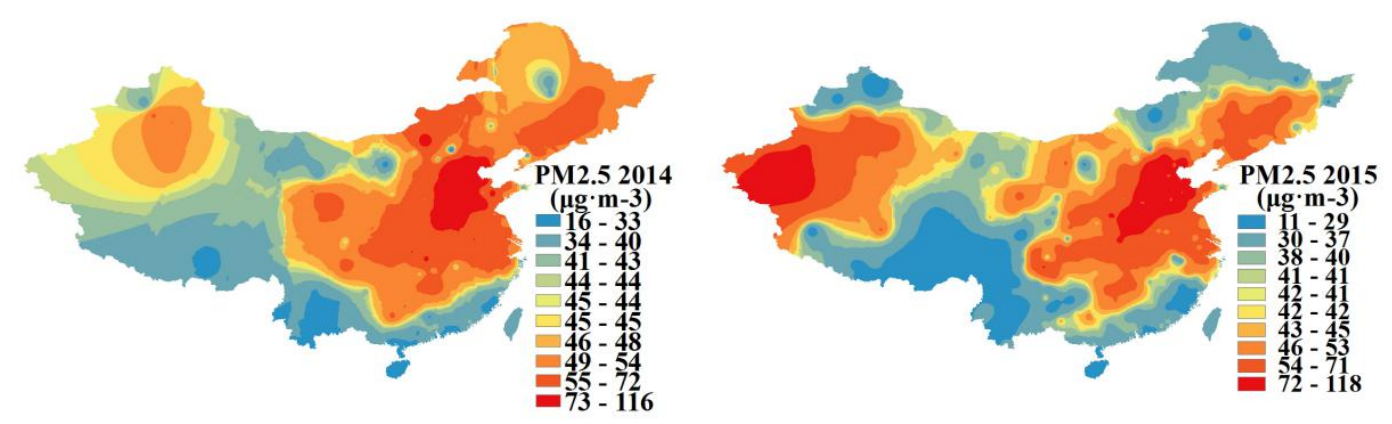

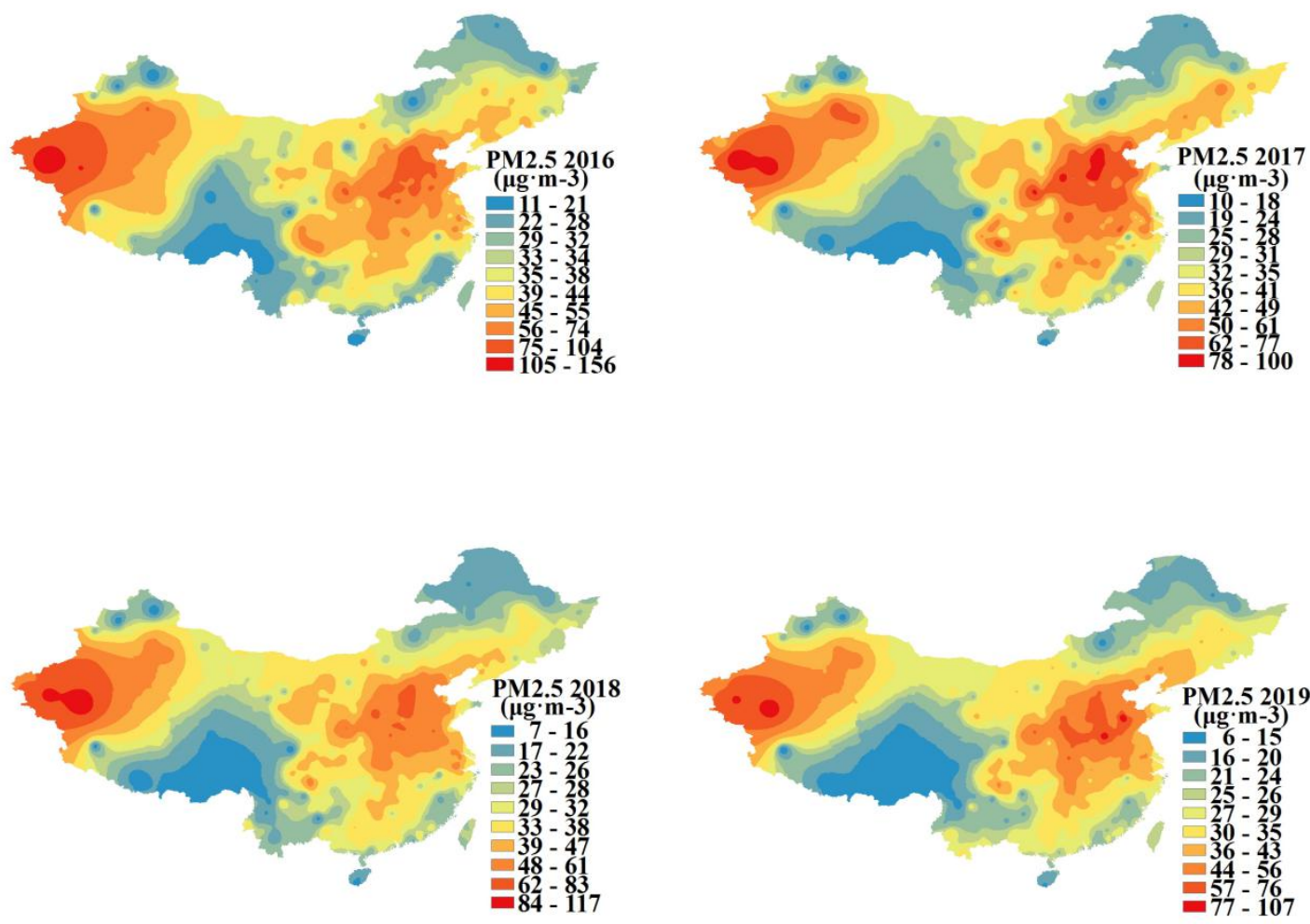

Figure 1. The spatial-temporal variation of the mass concentration of PM2.5 in mainland China from 2014 to 2019. 370 cities over the six-year period from 2014 to 2019, from which it can be seen that the proportions represented by some cities located in Beijing-Tianjin-Hebei region and Xinjiang region were about 5/6 and 1/6 respectively, with a lower proportion made up by some cities bordering Beijing-Tianjin-Hebei region. Among these records, Baoding and Xingtai, two cities situated in Beijing-Tianjin-Hebei region, combined with Kashi 112 and Hetian, two areas situated in Xinjiang region, ranked the top of the list five to six times in the six years. 
Table 1. Ten cities with higher mass concentration of PM2.5 among 370 cities

from 2014 to 2019.

\begin{tabular}{|c|c|c|c|c|c|c|c|c|}
\hline \multicolumn{3}{|c|}{2014 PM2.5 } & \multicolumn{3}{|c|}{2015 PM2.5 } & \multicolumn{3}{|c|}{2016 PM2.5 } \\
\hline City & $\begin{array}{c}\text { Belongs to } \\
\text { area }\end{array}$ & $\begin{array}{c}\text { Concentration } \\
\left(\mu \mathrm{g} \cdot \mathrm{m}^{-3}\right)\end{array}$ & City & $\begin{array}{c}\text { Belongs to } \\
\text { area }\end{array}$ & $\begin{array}{c}\text { Concentration } \\
/\left(\mu \mathrm{g} \cdot \mathrm{m}^{-3}\right)\end{array}$ & City & $\begin{array}{c}\text { Belongs to } \\
\text { area }\end{array}$ & $\begin{array}{c}\text { Concentration/ } \\
\left(\mu \mathrm{g} \cdot \mathrm{m}^{-3}\right)\end{array}$ \\
\hline Baoding $^{(5)}$ & Jingjinji & 117 & Kashi area ${ }^{(5)}$ & Xinjiang & 118 & Kashi area $^{(5)}$ & Xinjiang & 157 \\
\hline Xingtai $^{(6)}$ & Jingjinji & 105 & Baoding ${ }^{(5)}$ & Jingjinji & 106 & Hetian area ${ }^{(5)}$ & Xinjiang & 107 \\
\hline Handan $^{(4)}$ & Jing jinji & 100 & Dezhou $^{(2)}$ & $\begin{array}{r}\text { Shandong } \\
\text { (border } \\
\text { Jingjinji) }\end{array}$ & 101 & Shijiazhuang ${ }^{(4)}$ & Jingjinji & 94 \\
\hline Shijiazhuang $^{(4)}$ & Jingjinji & 99 & Xingtai ${ }^{(6)}$ & Jing jinji & 101 & Baoding $^{(5)}$ & Jing jinji & 92 \\
\hline Dezhou $^{(2)}$ & $\begin{array}{r}\text { Shandong } \\
\text { (border } \\
\text { Jing jinji) }\end{array}$ & 97 & Hengshui ${ }^{(4)}$ & Jing jinji & 98 & Akesu area ${ }^{(2)}$ & Xinjiang & 91 \\
\hline Hengshui $^{(4)}$ & jingjinji & 96 & Hetian area ${ }^{(5)}$ & Xinjiang & 98 & Hengshui $^{(4)}$ & Jing jinji & 87 \\
\hline Liaocheng $^{(3)}$ & $\begin{array}{r}\begin{array}{r}\text { Shandong } \\
\text { (border }\end{array} \\
\text { jingjinji) }\end{array}$ & 94 & Liaocheng $^{(3)}$ & $\begin{array}{r}\text { Shandong } \\
\text { (border } \\
\text { jingjinji) }\end{array}$ & 98 & Xingtai ${ }^{(6)}$ & Jing jinji & 87 \\
\hline Laiwu ${ }^{(2)}$ & $\begin{array}{r}\text { Shandong } \\
\text { (border } \\
\text { Jingjinji) }\end{array}$ & 90 & Zhengzhou $^{(1)}$ & $\begin{array}{c}\text { Henan } \\
\text { (border } \\
\text { jingjinji) }\end{array}$ & 95 & Liaocheng $^{(3)}$ & $\begin{array}{r}\text { Shandong } \\
\text { (border } \\
\text { jing jinji) }\end{array}$ & 86 \\
\hline Tangshan $^{(1)}$ & Jingjinji & 90 & $\mathrm{Heze}^{(1)}$ & $\begin{array}{r}\text { Shandong } \\
\text { (border } \\
\text { jingjinji) }\end{array}$ & 94 & Jiazuo $^{(1)}$ & $\begin{array}{c}\text { Henan } \\
\text { (border } \\
\text { jing jinji) }\end{array}$ & 85 \\
\hline Langfang $^{(1)}$ & Jingjinji & 89 & Xinxiang $^{(1)}$ & $\begin{array}{c}\text { Henan } \\
\text { (border } \\
\text { jingjinji) }\end{array}$ & 93 & Anyang $^{(4)}$ & $\begin{array}{c}\text { Henan } \\
\text { (border } \\
\text { jing jinji) }\end{array}$ & 85 \\
\hline
\end{tabular}




\begin{tabular}{|c|c|c|c|c|c|c|c|c|}
\hline \multicolumn{3}{|c|}{2017 PM2.5 } & \multicolumn{3}{|c|}{2018 PM 2.5} & \multicolumn{3}{|c|}{2019 PM2.5 } \\
\hline City & $\begin{array}{c}\text { Belongs to } \\
\text { area }\end{array}$ & $\begin{array}{c}\text { Concentration/ } \\
\left(\mu \mathrm{g} \cdot \mathrm{m}^{-3}\right)\end{array}$ & City & $\begin{array}{c}\text { Belongs to } \\
\text { area }\end{array}$ & $\begin{array}{c}\text { Concentration } \\
/\left(\mu \mathrm{g} \cdot \mathrm{m}^{-3}\right)\end{array}$ & City & $\begin{array}{c}\text { Belongs to } \\
\text { area }\end{array}$ & $\begin{array}{c}\text { Concentration } \\
/\left(\mu \mathrm{g} \cdot \mathrm{m}^{-3}\right)\end{array}$ \\
\hline Kashi area ${ }^{(5)}$ & Xinjiang & 100 & Hetian area ${ }^{(5)}$ & Xinjiang & 117 & Hetian area ${ }^{(5)}$ & Xinjiang & 108 \\
\hline Hetian area $^{(5)}$ & Xinjiang & 92 & Kashi $\operatorname{area}^{(5)}$ & Xinjiang & 94 & Laiwu $^{(2)}$ & $\begin{array}{c}\text { Shandong } \\
\text { (border } \\
\text { jingjinji) }\end{array}$ & 106 \\
\hline Handan $^{(4)}$ & Jingjinji & 86 & Akesu area $^{(2)}$ & Xinjiang & 74 & Shangqiu ${ }^{(1)}$ & $\begin{array}{c}\text { Henan } \\
\text { (border } \\
\text { jingjinji) }\end{array}$ & 94 \\
\hline Baoding ${ }^{(5)}$ & Jingjinji & 84 & Anyang ${ }^{(4)}$ & $\begin{array}{c}\text { Henan } \\
\text { (border } \\
\text { jingjinji })\end{array}$ & 69 & Kashi $\operatorname{area}^{(5)}$ & Xinjiang & 85 \\
\hline Linfen $^{(3)}$ & $\begin{array}{c}\text { shanxi } \\
\quad \text { border } \\
\text { Jingjinji) }\end{array}$ & 82 & Shi jiazhuang ${ }^{(4)}$ & Jingjinji & 68 & Anyang ${ }^{(4)}$ & $\begin{array}{c}\text { Henan } \\
\text { (border } \\
\text { jingjinji) }\end{array}$ & 67 \\
\hline Anyang ${ }^{(4)}$ & $\begin{array}{c}\text { Henan } \\
\text { (border } \\
\text { jingjinji) }\end{array}$ & 82 & $\operatorname{Linfen}^{(3)}$ & $\begin{array}{c}\text { shanxi } \\
\text { (border } \\
\text { Jingjinji) }\end{array}$ & 67 & Handan $^{(4)}$ & Jingjinji & 63 \\
\hline Shi jiazhuang ${ }^{(4)}$ & Jingjinji & 82 & Xingtai ${ }^{(6)}$ & Jing jinji & 66 & Xingtai ${ }^{(6)}$ & Jingjinji & 62 \\
\hline Xingtai $^{(6)}$ & Jingjinji & 81 & Handan ${ }^{(4)}$ & Jingjinji & 65 & Luoyang $^{(1)}$ & $\begin{array}{c}\text { Henan } \\
\text { (border } \\
\text { jing jinji) }\end{array}$ & 60 \\
\hline Xianyang ${ }^{(1)}$ & $\begin{array}{c}\text { shanxi } \\
\quad \text { (border } \\
\text { Jingjinji) }\end{array}$ & 79 & Baoding $^{(5)}$ & Jingjinji & 65 & $\operatorname{Linfen}^{(3)}$ & $\begin{array}{c}\text { shanxi } \\
\quad(\text { border } \\
\text { Jingjinji) }\end{array}$ & 59 \\
\hline Hengshui ${ }^{(4)}$ & Jingjinji & 77 & Xuzhou $^{(1)}$ & Jiangsu & 62 & Puyang ${ }^{(1)}$ & $\begin{array}{c}\text { Henan } \\
\text { (border } \\
\text { jingjinji) }\end{array}$ & 59 \\
\hline
\end{tabular}

$\mathrm{CO}, \mathrm{NO} 2, \mathrm{SO} 2$, but it of $\mathrm{O} 3$ would indicate a different trend compared with AQI. 
132 with time. The temporal variation of AQI had a similar trend to that of the above PM2.5
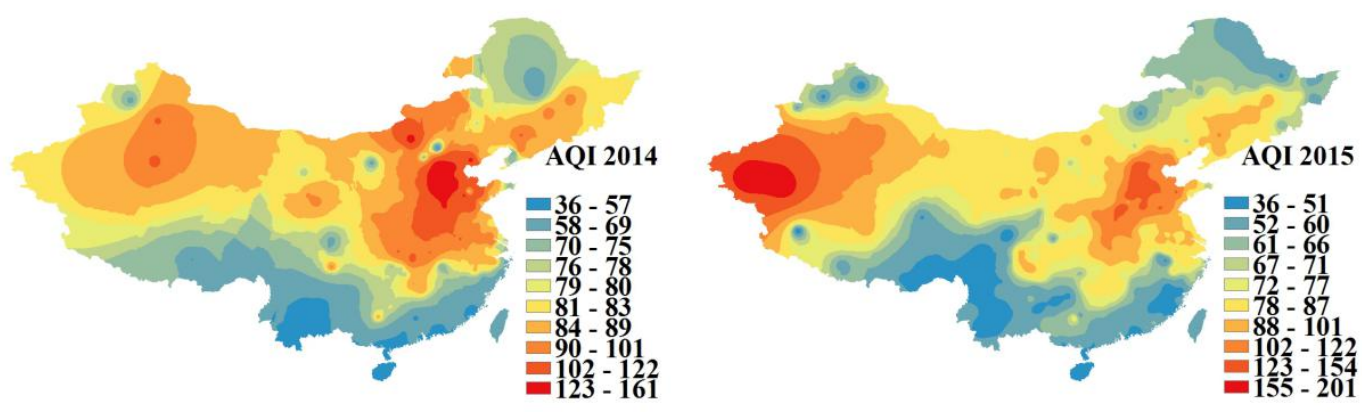

139
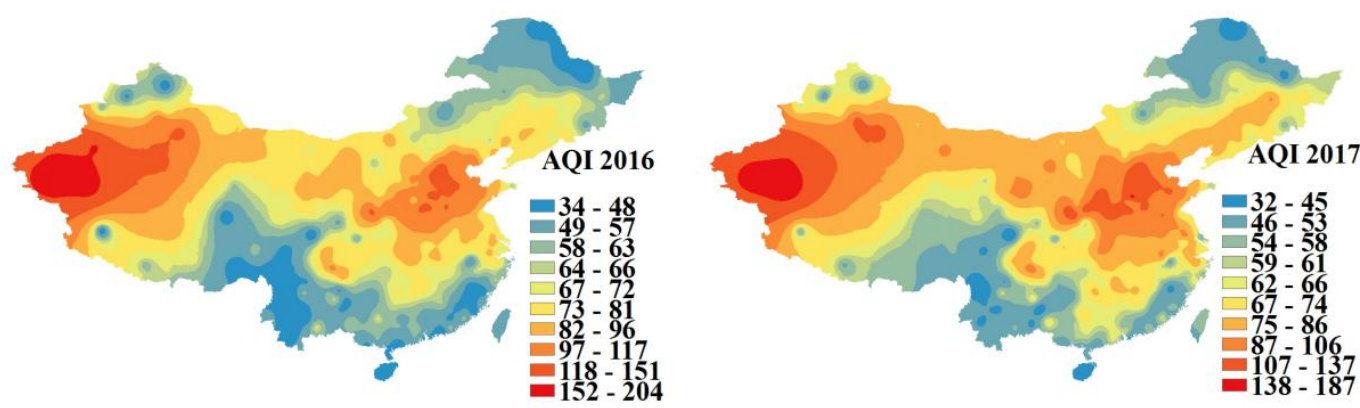
141
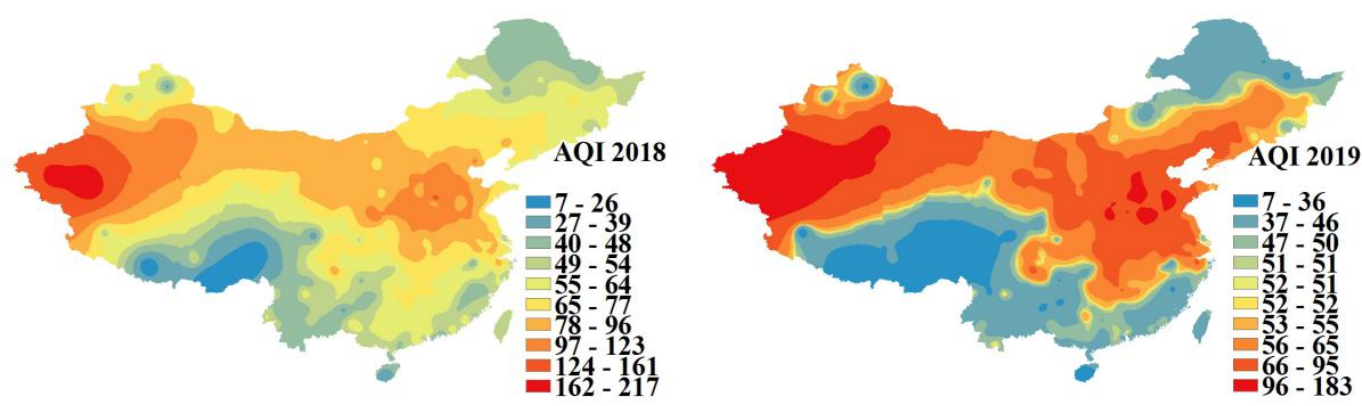

Figure 2. The spatial-temporal variation of the annual average AQI in mainland China from 2014 to 2019

According to Table 2 which indicates the ten cities with higher mass concentration of AQI among 370 cities over the six-year period from 2014 to 2019, it can be seen that the proportions represented by some cities located in Beijing-TianjinHebei region and Xinjiang region were almost the same, at about $1 / 2$, with a lower proportion made up by some cities bordering Beijing-Tianjin-Hebei region. Among these records, Baoding, Xingtai and Handan, three cities situated in Beijing-TianjinHebei region, combined with Kashi, Hetian, Aksu and Kezhou, four areas situated in Xinjiang region, ranked the top of the list four to five times in the six years.

Table 2. Ten cities with higher annual average AQI and their affiliated regions from 2014 to 2019. 
bioRxiv preprint doi: https://doi.org/10.1101/2019.12.20.884346; this version posted December 20, 2019. The copyright holder for this preprint (which was not certified by peer review) is the author/funder, who has granted bioRxiv a license to display the preprint in perpetuity. It is made available under aCC-BY 4.0 International license.

\begin{tabular}{|c|c|c|c|c|c|c|c|c|}
\hline \multicolumn{3}{|c|}{2014 AQI } & \multicolumn{3}{|c|}{2015 AQI } & \multicolumn{3}{|c|}{2016 AQI } \\
\hline City & $\begin{array}{c}\text { Belongs to } \\
\text { area }\end{array}$ & AQI & City & $\begin{array}{l}\text { Belongs to } \\
\text { area }\end{array}$ & AQI & City & $\begin{array}{l}\text { Belongs to } \\
\text { area }\end{array}$ & AQI \\
\hline Baoding $^{(4)}$ & Jingjinji & 162 & Hetian $\operatorname{area}^{(5)}$ & Xinjiang & 201 & Kashi area $^{(5)}$ & Xinjiang & 205 \\
\hline Xingtai $^{(5)}$ & Jingjinji & 146 & Kashi area ${ }^{(5)}$ & Xinjiang & 198 & 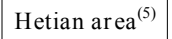 & Xinjiang & 184 \\
\hline Hengshui $^{(3)}$ & Jingjinji & 143 & Akesu area ${ }^{(5)}$ & Xinjiang & 148 & Akesu area ${ }^{(5)}$ & Xinjiang & 155 \\
\hline Handan $^{(4)}$ & Jingjinji & 140 & Baoding $^{(4)}$ & Jingjinji & 144 & Kezhou area $^{(5)}$ & Xinjiang & 147 \\
\hline Shijiazhuang ${ }^{(4)}$ & Jingjinji & 138 & Hengshui $^{(3)}$ & Jingjinji & 141 & Kuerle $^{(3)}$ & Xinjiang & 133 \\
\hline Dezhou $^{(3)}$ & $\begin{array}{l}\text { Shandong } \\
\text { (border } \\
\text { Jingjinji) }\end{array}$ & 131 & Dezhou $^{(3)}$ & $\begin{array}{l}\text { Shandong } \\
\quad \text { (border } \\
\text { Jingjinji) }\end{array}$ & 139 & Shijiazhuang $^{(3)}$ & Jingjinji & 131 \\
\hline Liaozheng $^{(2)}$ & $\begin{array}{l}\text { Shandong } \\
\text { (border } \\
\text { J ingjinji) }\end{array}$ & 130 & Xingtai $^{(5)}$ & Jingjinji & 138 & Baoding $^{(4)}$ & Jingjinji & 128 \\
\hline Langfang $^{(1)}$ & Jingjinji & 127 & Kezhou area ${ }^{(5)}$ & Xinjiang & 137 & Tulufan area ${ }^{(4)}$ & Xinjiang & 127 \\
\hline Tangshan $^{(1)}$ & J ingjinji & 126 & Zhengzhou $^{(1)}$ & $\begin{array}{c}\text { Henan } \\
\text { (border } \\
\text { jingjinji ) }\end{array}$ & 135 & Hengshui $^{(3)}$ & Jingjinji & 126 \\
\hline Heze $^{(1)}$ & $\begin{array}{l}\text { Shandong } \\
\text { (border } \\
\text { Jingjinji) }\end{array}$ & 125 & Liaozheng $^{(2)}$ & $\begin{array}{l}\text { Shandong } \\
\quad \text { (border } \\
\text { Jingjinji) }\end{array}$ & 134 & Dezhou $^{(3)}$ & $\begin{array}{l}\text { Shandong } \\
\text { (border } \\
\text { Jingjinji) }\end{array}$ & 123 \\
\hline \multicolumn{3}{|c|}{2017 AQI } & \multicolumn{3}{|c|}{2018 AQI } & \multicolumn{3}{|c|}{2019 AQI } \\
\hline City & $\begin{array}{l}\text { Belongs to } \\
\text { area }\end{array}$ & AQI & City & $\begin{array}{l}\text { Belongs to } \\
\text { area }\end{array}$ & AQI & City & $\begin{array}{l}\text { Belongs to } \\
\text { area }\end{array}$ & AQI \\
\hline Hetian area ${ }^{(5)}$ & Xinjiang & 187 & Hetian area ${ }^{(5)}$ & Xinjiang & 217 & Hetian area ${ }^{(5)}$ & Xinjiang & 184 \\
\hline Kashi area $^{(5)}$ & Xinjiang & 162 & Kashi area $^{(5)}$ & Xinjiang & 180 & Kashi area $^{(5)}$ & Xinjiang & 152 \\
\hline Akesu area ${ }^{(5)}$ & Xinjiang & 135 & Kezhou area ${ }^{(5)}$ & Xinjiang & 155 & Laiwu $^{(1)}$ & $\begin{array}{l}\text { Shandong } \\
\text { (border } \\
\text { Jingjinji) }\end{array}$ & 142 \\
\hline Handan $^{(4)}$ & Jingjinji & 126 & Akesu area ${ }^{(5)}$ & Xinjiang & 153 & Shangqiu $^{(1)}$ & $\begin{array}{c}\text { Henan } \\
\text { (border } \\
\text { jingjinji) }\end{array}$ & 134 \\
\hline Anyang $^{(3)}$ & $\begin{array}{c}\text { Henan } \\
\text { (border } \\
\text { jingjinji) }\end{array}$ & 123 & Tulufan area ${ }^{(4)}$ & Xinjiang & 122 & Akesu area $^{(5)}$ & Xinjiang & 120 \\
\hline Xingtai $^{(5)}$ & J ingjinji & 122 & Kuerle $^{(3)}$ & Xinjiang & 121 & Kezhou area $^{(5)}$ & Xinjiang & 118 \\
\hline Baoding $^{(4)}$ & Jingjinji & 122 & Anyang $^{(3)}$ & $\begin{array}{c}\text { Henan } \\
\text { (border } \\
\text { jingjinji) }\end{array}$ & 115 & Kuerle $^{(3)}$ & Xinjiang & 116 \\
\hline Shijiazhuang $^{(3)}$ & J ingjinji & 122 & Xingtai $^{(5)}$ & J ingjinji & 114 & Tulufan area ${ }^{(4)}$ & Xinjiang & 112 \\
\hline Xianyang $^{(2)}$ & $\begin{array}{c}\text { shanxi } \\
\text { (border } \\
\text { Jingjinji ) }\end{array}$ & 121 & Handan $^{(4)}$ & Jingjinji & 114 & Anyang $^{(3)}$ & $\begin{array}{c}\text { Henan } \\
\text { (border } \\
\text { jingjinji) }\end{array}$ & 106 \\
\hline Kezhou area ${ }^{(5)}$ & Xinjiang & 120 & Wujiaqu $^{(1)}$ & Xinjiang & 113 & Handan $^{(4)}$ & Jingjinji & 102 \\
\hline Tulufan area ${ }^{(4)}$ & Xinjiang & 120 & Xianyang $^{(2)}$ & $\begin{array}{c}\text { Shanxi } \\
\text { (border } \\
\text { Jingjinji) }\end{array}$ & 112 & Xingtai $^{(5)}$ & Jingjinji & 100 \\
\hline
\end{tabular}


161

162

northwest of mainland China showed higher mass concentration (including the highest mass concentration) of PM10 from the year 2014 to 2019. There was a similar trend between the spatial variation of PM10 and it of the above PM2.5 and AQI and the following $\mathrm{CO}, \mathrm{NO} 2$ and $\mathrm{SO} 2$, but it of the following $\mathrm{O} 3$ would indicate a different trend compared with PM10. The highest mass concentration of PM10 was $196 \mu \mathrm{g} \cdot \mathrm{m}-3$, $340 \mu \mathrm{g} \cdot \mathrm{m}-3, \quad 434 \mu \mathrm{g} \cdot \mathrm{m}-3,317 \mu \mathrm{g} \cdot \mathrm{m}-3, \quad 432 \mu \mathrm{g} \cdot \mathrm{m}-3,334 \mu \mathrm{g} \cdot \mathrm{m}-3$ respectively in every separated year between 2014 and 2019. The highest PM10 in the year 2016 and 2014 were the highest and lowest among the six years, reaching $434 \mu \mathrm{g} \cdot \mathrm{m}-3$ and $196 \mu \mathrm{g} \cdot \mathrm{m}$ 3 respectively. In addition, this index represented increase and then decrease, followed by an increase and decrease again, a kind of S-shaped change with time. The temporal variation of PM10 had a similar trend to that of the above PM2.5 and AQI. On the other hand, from the perspective of the annual highest mass concentration of PM10, it can be analyzed that the atmospheric condition of mainland China had not been authentically improved until the year 2019.
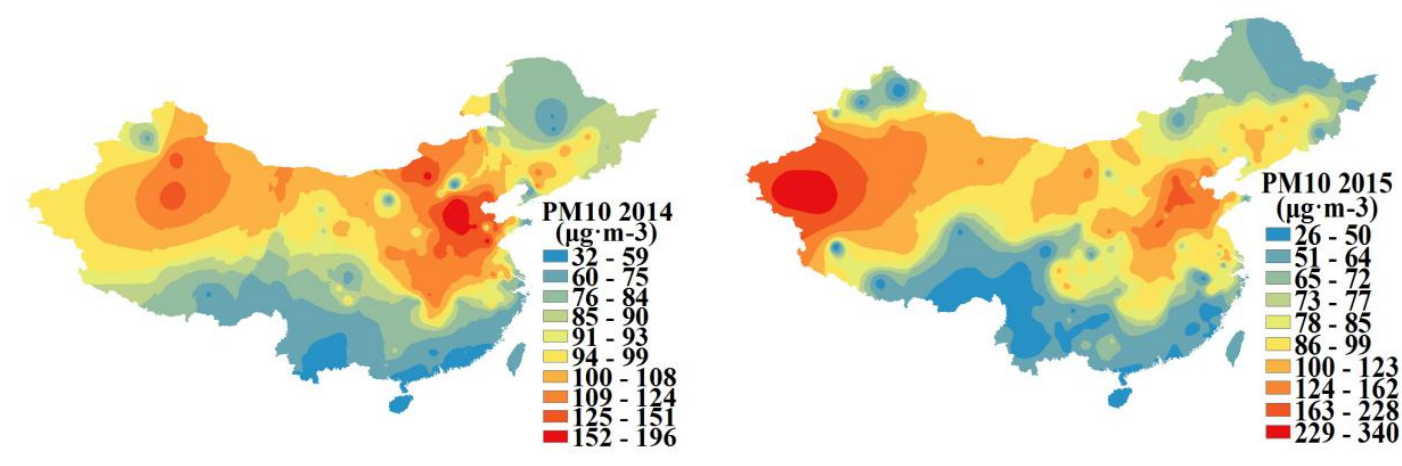

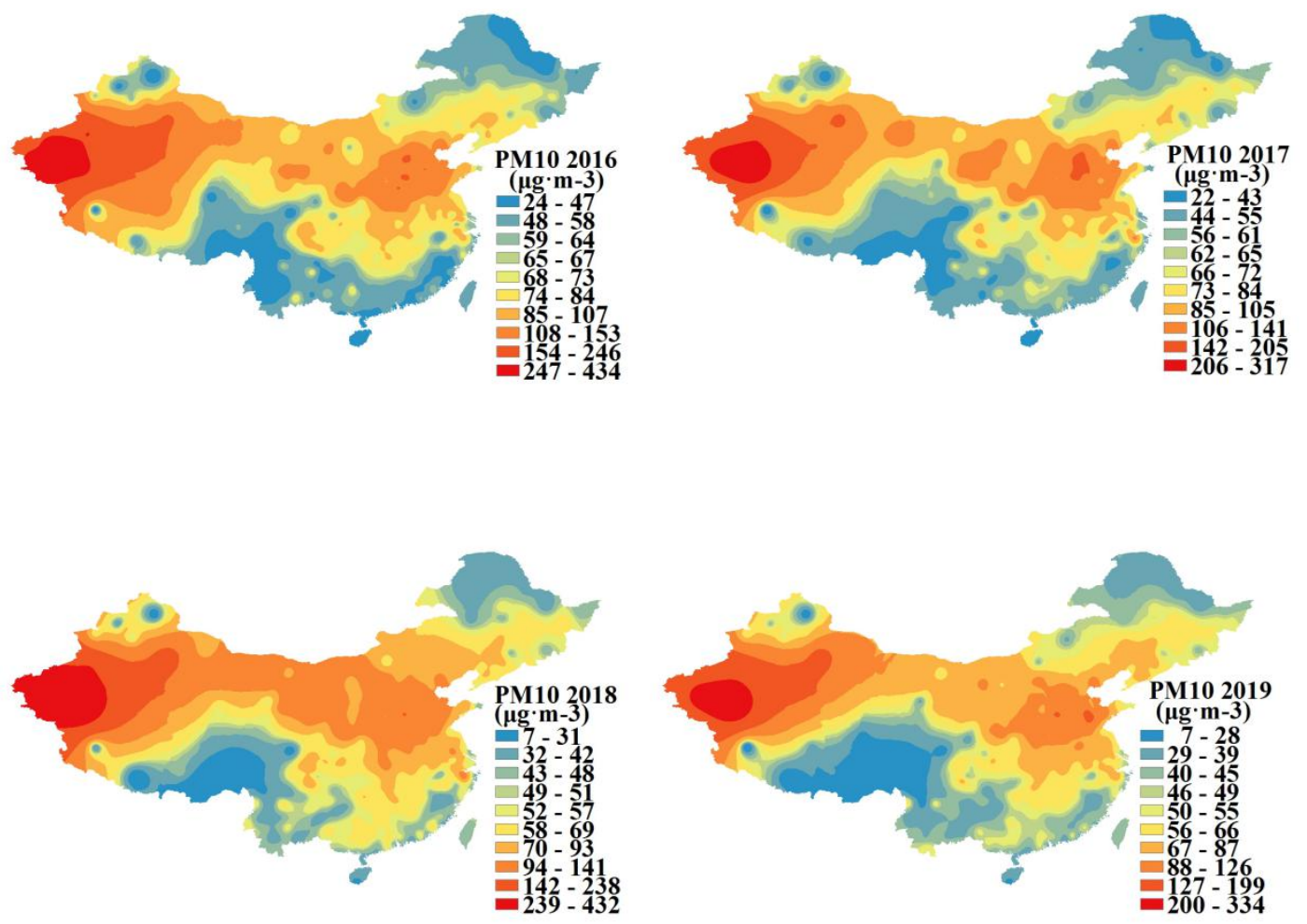

Figure 3. The spatial-temporal variation of the annual average mass concentration of PM10 in mainland China from 2014 to 2019 
bioRxiv preprint doi: https://doi.org/10.1101/2019.12.20.884346; this version posted December 20, 2019. The copyright holder for this preprint (which was not certified by peer review) is the author/funder, who has granted bioRxiv a license to display the preprint in perpetuity. It is made available under aCC-BY 4.0 International license.

Table 3. Ten cities with higher annual average mass concentration of PM10 and

\begin{tabular}{|c|c|c|c|c|c|c|c|c|}
\hline \multicolumn{3}{|c|}{2014 PM10 } & \multicolumn{3}{|c|}{2015 PM10 } & \multicolumn{3}{|c|}{2016 PM 10} \\
\hline City & $\begin{array}{l}\text { Belongs to } \\
\text { area }\end{array}$ & $\begin{array}{c}\text { Concentration } \\
/\left(\mu \mathrm{g} \cdot \mathrm{m}^{-3}\right)\end{array}$ & City & Belongs to area & $\begin{array}{c}\text { Concentration } \\
/\left(\mu \mathrm{g} \cdot \mathrm{m}^{-3}\right)\end{array}$ & City & Belongs to area & $\begin{array}{c}\text { Concentration } \\
/\left(\mu \mathrm{g} \cdot \mathrm{m}^{-3}\right)\end{array}$ \\
\hline Baoding $^{(2)}$ & Jingjinji & 198 & Hetian area ${ }^{(5)}$ & Xinjiang & 341 & Kasha area ${ }^{(5)}$ & Xinjiang & 435 \\
\hline Xingtai & J ingjinji & 187 & Kasha area ${ }^{(5)}$ & Xinjiang & 308 & Hetian area ${ }^{(5)}$ & Xinjiang & 283 \\
\hline Hengshui $^{(2)}$ & Jingjinji & 176 & Akesu area ${ }^{(5)}$ & Xinjiang & 217 & Akesu area ${ }^{(5)}$ & Xinjiang & 246 \\
\hline Handan $^{(6)}$ & J ingjinji & 174 & Kezhou $^{(5)}$ & Xinjiang & 186 & Kezhou $^{(5)}$ & Xinjiang & 217 \\
\hline Shijiazhuang $^{(3)}$ & Jingjinji & 172 & Hengshui ${ }^{(2)}$ & Jingjinji & 176 & Kuerle ${ }^{(5)}$ & Xinjiang & 210 \\
\hline Liaocheng $^{(2)}$ & $\begin{array}{l}\text { Shandong } \\
\text { (border } \\
\text { jingjinji) }\end{array}$ & 165 & Baoding $^{(2)}$ & Jingjinji & 175 & Tulufan area ${ }^{(4)}$ & Xinjiang & 170 \\
\hline Linyi $^{(1)}$ & $\begin{array}{l}\text { Shandong } \\
\text { (border } \\
\text { jingjinji) }\end{array}$ & 163 & Xingtai $^{(1)}$ & J ingjinji & 172 & Shijiazhuang ${ }^{(3)}$ & J ingjinji & 161 \\
\hline$Z_{i b o}^{(1)}$ & $\begin{array}{l}\text { Shandong } \\
\text { (border } \\
\text { jingjinji) }\end{array}$ & 161 & Handan $^{(6)}$ & Jingjinji & 167 & Dezhou $^{(2)}$ & $\begin{array}{l}\text { Shandong } \\
\text { (border } \\
\text { jingjinji) }\end{array}$ & 152 \\
\hline $\operatorname{Jinan}^{(1)}$ & $\begin{array}{l}\text { Shandong } \\
\text { (border } \\
\text { jingjinji) }\end{array}$ & 158 & Zhengzhou $^{(1)}$ & $\begin{array}{c}\text { Henan (border } \\
\text { jingjinji) }\end{array}$ & 166 & Handan $^{(6)}$ & $\mathrm{J}$ ingjinji & 151 \\
\hline Kuerle $e^{(5)}$ & Xinjiang & 152 & Dezhou $^{(2)}$ & $\begin{array}{l}\text { Shandong } \\
\text { (border } \\
\text { jingjinji) }\end{array}$ & 165 & Liaocheng $^{(2)}$ & $\begin{array}{l}\text { Shandong } \\
\text { (border } \\
\text { jingjinji) }\end{array}$ & 150 \\
\hline
\end{tabular}

\begin{tabular}{|c|c|c|c|c|c|c|c|c|}
\hline \multicolumn{3}{|c|}{2017 PM10 } & \multicolumn{3}{|c|}{2018 PM10 } & \multicolumn{3}{|c|}{2019 PM 10} \\
\hline City & $\begin{array}{c}\text { Belongs to } \\
\text { area }\end{array}$ & $\begin{array}{c}\text { Concentration } \\
/\left(\mu \mathrm{g} \cdot \mathrm{m}^{-3}\right)\end{array}$ & City & Belongs to area & $\begin{array}{c}\text { Concentration } \\
/\left(\mu \mathrm{g} \cdot \mathrm{m}^{-3}\right)\end{array}$ & City & Belongs to area & $\begin{array}{c}\text { Concentration } \\
/\left(\mu \mathrm{g} \cdot \mathrm{m}^{-3}\right)\end{array}$ \\
\hline Hetian area ${ }^{(5)}$ & Xinjiang & 318 & Hetian area ${ }^{(5)}$ & Xinjiang & 432 & Hetian area ${ }^{(5)}$ & Xinjiang & 335 \\
\hline Kasha area ${ }^{(5)}$ & Xinjiang & 248 & Kasha area ${ }^{(5)}$ & Xinjiang & 331 & Kasha area ${ }^{(5)}$ & Xinjiang & 229 \\
\hline Akesu area ${ }^{(5)}$ & Xinjiang & 198 & Kezhou $^{(5)}$ & Xinjiang & 291 & Kezhou $^{(5)}$ & Xinjiang & 182 \\
\hline Tulufan area ${ }^{(4)}$ & Xinjiang & 158 & Akesu area ${ }^{(5)}$ & Xinjiang & 256 & Akesu area ${ }^{(5)}$ & Xinjiang & 181 \\
\hline Handan $^{(6)}$ & Jingjinji & 157 & Tulufan area ${ }^{(4)}$ & Xinjiang & 180 & Kuerle $^{(5)}$ & Xinjiang & 171 \\
\hline Kezhou $^{(5)}$ & Xinjiang & 154 & Kuerle $^{(5)}$ & Xinjiang & 171 & Laiwu $^{(1)}$ & $\begin{array}{l}\text { Shandong } \\
\text { (border } \\
\text { jingjinji) }\end{array}$ & 168 \\
\hline Shijiazhuang ${ }^{(3)}$ & J ingjinji & 153 & Wujiaqu $^{(1)}$ & Xinjiang & 136 & Tulufan area ${ }^{(4)}$ & Xinjiang & 164 \\
\hline Xingtai $^{(2)}$ & Jingjinji & 152 & Handan $^{(6)}$ & Jingjinji & 136 & Handan $^{(6)}$ & Jingjinji & 124 \\
\hline Pingdu ${ }^{(1)}$ & $\begin{array}{r}\text { Shandong } \\
\text { (border } \\
\text { jingjinji) }\end{array}$ & 150 & Xingtai $^{(2)}$ & Jingjinji & 136 & Shangqiu ${ }^{(1)}$ & $\begin{array}{c}\text { Henan (border } \\
\text { jingjinji) }\end{array}$ & 124 \\
\hline Kuerle $^{(5)}$ & Xinjiang & 142 & Weinan $^{(1)}$ & $\begin{array}{c}\text { Shanxi } \\
\text { (border } \\
\text { Jingjinji) }\end{array}$ & 135 & Zaozhuang $^{(1)}$ & $\begin{array}{c}\text { Shandong } \\
\text { (border } \\
\text { jingjinji) }\end{array}$ & 117 \\
\hline
\end{tabular}


198 and its surrounding region in the northeast of mainland China and Xinjiang region in the northwest of mainland China showed higher mass concentration (including the highest mass concentration) of CO from the year 2014 to 2019. There was a similar trend between the spatial variation of $\mathrm{CO}$ and it of $\mathrm{PM} 2.5, \mathrm{PM} 10, \mathrm{AQI}, \mathrm{NO} 2$ and $\mathrm{SO} 2$ acquired in the above and following contents, but it of the following $\mathrm{O} 3$ would indicate a different trend compared with $\mathrm{CO}$. The highest mass concentration of $\mathrm{CO}$ was 2.2 $\mu \mathrm{g} \cdot \mathrm{m}-3, \quad 2.5 \mu \mathrm{g} \cdot \mathrm{m}-3, \quad 2.4 \mu \mathrm{g} \cdot \mathrm{m}-3,2 \mu \mathrm{g} \cdot \mathrm{m}-3, \quad 1.7 \mu \mathrm{g} \cdot \mathrm{m}-3,1.1 \mu \mathrm{g} \cdot \mathrm{m}-3$ respectively in every separated year between 2014 and 2019. The highest mass concentration of CO in the year 2015 and 2019 were the highest $(2.5 \mu \mathrm{g} \cdot \mathrm{m}-3)$ and lowest $(1.1 \mu \mathrm{g} \cdot \mathrm{m}-3)$ among the six years. It also shows that this indicator had experienced a dramatic decline by the year 2019, which was less than the half of the figure in 2015. On the other hand, the temporal variation of $\mathrm{CO}$ witnessed a similar trend to that of the following $\mathrm{NO} 2$ and SO2. In addition, what is worth pointing is that extraordinarily high concentrations arose in Xinjiang region from 2018 to 2019. From the perspective of the annual highest mass concentration of $\mathrm{CO}$, it can be analyzed that the atmospheric condition of mainland China had not been authentically improved until the year 2019 . 

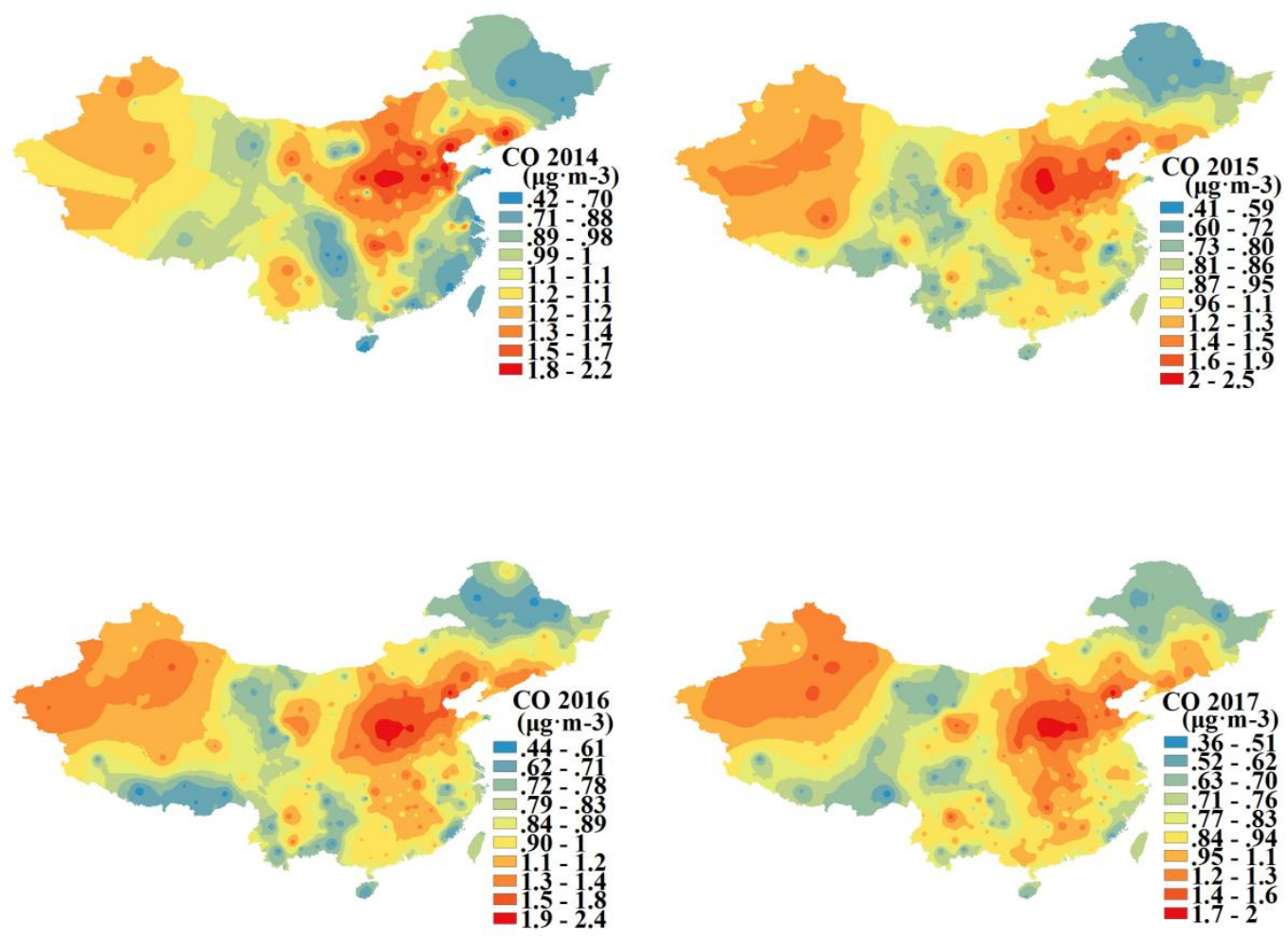

Figure 4. The spatial-temporal variation of the mass concentration of $\mathrm{CO}$ in
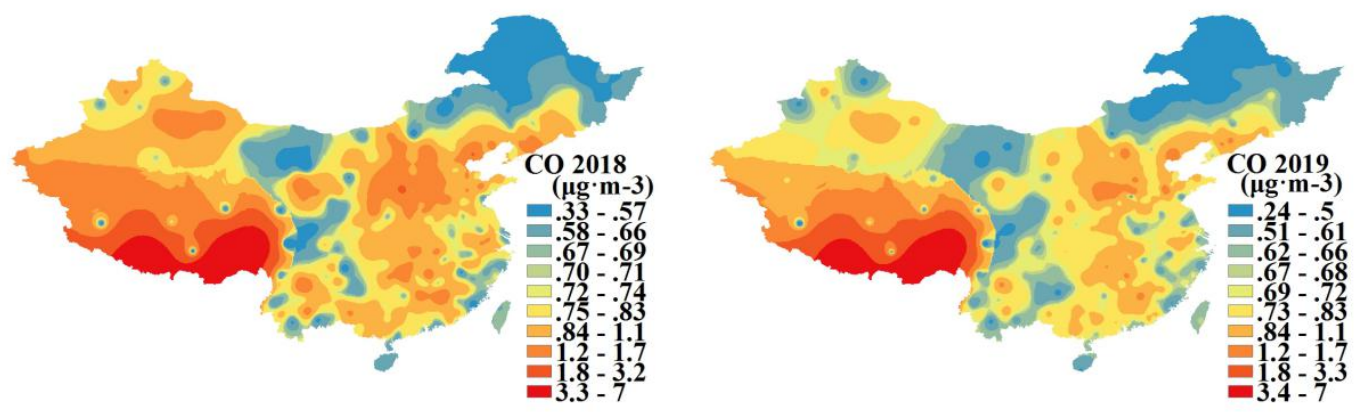
bioRxiv preprint doi: https://doi.org/10.1101/2019.12.20.884346; this version posted December 20, 2019. The copyright holder for this preprint (which was not certified by peer review) is the author/funder, who has granted bioRxiv a license to display the preprint in perpetuity. It is made available under aCC-BY 4.0 International license.

224 areas like Shandong and Henan represented 1/2. To be more specific, Linfen and

225 Changzhi, two cities situated in Shanxi province, combined with Anyang, a city situated

227 consecutively in the six years.

Table 4. Ten cities with higher annual average mass concentration of $\mathrm{CO}$ and

their affiliated regions from 2014 to 2019

\begin{tabular}{|c|c|c|c|c|c|c|c|c|}
\hline \multicolumn{3}{|c|}{$2014 \mathrm{CO}$} & \multicolumn{3}{|c|}{$2015 \mathrm{CO}$} & \multicolumn{3}{|c|}{$2016 \mathrm{CO}$} \\
\hline City & $\begin{array}{l}\text { Belongs to } \\
\text { area }\end{array}$ & $\begin{array}{c}\text { Concentration } \\
/\left(\mu \mathrm{g} \cdot \mathrm{m}^{-3}\right)\end{array}$ & City & $\begin{array}{c}\begin{array}{c}\text { Belongs to } \\
\text { area }\end{array} \\
\end{array}$ & $\begin{array}{c}\text { Concentration } \\
/\left(\mu \mathrm{g} \cdot \mathrm{m}^{-3}\right)\end{array}$ & City & $\begin{array}{c}\text { Belongs to } \\
\text { area }\end{array}$ & $\begin{array}{c}\text { Concentration } \\
/\left(\mu \mathrm{g} \cdot \mathrm{m}^{-3}\right)\end{array}$ \\
\hline Penglai(2) & $\begin{array}{l}\text { Shandong } \\
\text { (border } \\
\text { Jingjinji) }\end{array}$ & 2.25 & Lvliang(1) & $\begin{array}{c}\text { Shanxi } \\
\text { (border } \\
\text { Jingjinji) }\end{array}$ & 2.75 & Linfen(6) & $\begin{array}{c}\text { Shanxi } \\
\text { (border } \\
\text { Jingjinji) }\end{array}$ & 2.52 \\
\hline Linfen(6) & $\begin{array}{c}\text { Shanxi } \\
\text { (border } \\
\text { Jingjinji) }\end{array}$ & 2.20 & Linfen $(6)$ & $\begin{array}{c}\text { Shanxi } \\
\text { (border } \\
\text { Jingjinji ) }\end{array}$ & 2.36 & Tangshan(6) & J ingjinji & 2.27 \\
\hline Binzhou(2) & $\begin{array}{c}\text { Shandong } \\
\text { (border } \\
\text { Jingjinji) }\end{array}$ & 2.13 & Tangshan(6) & Jingjinji & 2.13 & Anyang(5) & $\begin{array}{c}\text { Henan } \\
\text { (border } \\
\text { jingjinji ) }\end{array}$ & 2.17 \\
\hline Zibo(4) & $\begin{array}{l}\text { Shandong } \\
\text { (border } \\
\text { Jingjinji) }\end{array}$ & 2.11 & Zibo(4) & $\begin{array}{c}\text { Shandong } \\
\text { (border } \\
\text { Jingjinji) }\end{array}$ & 2.11 & Yuncheng(3) & $\begin{array}{c}\text { Shanxi } \\
\text { (border } \\
\text { Jingjinji) }\end{array}$ & 2.06 \\
\hline Benxi(1) & $\begin{array}{r}\text { Liaoning } \\
\text { (border } \\
\text { Jingjinji) }\end{array}$ & 2.09 & Binzhou(2) & $\begin{array}{r}\text { Shandong } \\
\quad \text { border } \\
\text { J ingjinji) }\end{array}$ & 2.10 & Jincheng $(4)$ & $\begin{array}{c}\text { Shanxi } \\
\text { (border } \\
\text { Jingjinji) }\end{array}$ & 2.00 \\
\hline Tangshan(6) & J ingjinji & 2.06 & Naqu area(1) & Xinjiang & 2.09 & Luoyang(1) & $\begin{array}{c}\text { Henan } \\
\text { (border } \\
\text { jingjinji) }\end{array}$ & 2.00 \\
\hline Changzhi(5) & $\begin{array}{c}\text { Shanxi } \\
\text { (border } \\
\text { Jingjinji) }\end{array}$ & 2.05 & Anyang(5) & $\begin{array}{c}\text { Henan } \\
\text { (border } \\
\text { jingjinji) }\end{array}$ & 2.06 & Changzhi(5) & $\begin{array}{c}\text { Shanxi } \\
\text { (border } \\
\text { Jingjinji) }\end{array}$ & 2.00 \\
\hline Liaocheng(1) & $\begin{array}{c}\text { Shandong } \\
\text { (border } \\
\text { Jingjinji) }\end{array}$ & 2.00 & Zhoukou(1) & $\begin{array}{c}\text { Henan } \\
\text { (border } \\
\text { jingjinji) }\end{array}$ & 2.02 & Hebi(2) & $\begin{array}{c}\text { Henan } \\
\text { (border } \\
\text { jingjinji) }\end{array}$ & 1.96 \\
\hline Yanan(1) & $\begin{array}{c}\text { Shanxi } \\
\text { (border } \\
\text { Jingjinji ) }\end{array}$ & 1.93 & Yuncheng(3) & $\begin{array}{c}\text { Shanxi } \\
\text { (border } \\
\text { Jingjinji ) }\end{array}$ & 1.96 & Zibo(4) & $\begin{array}{l}\text { Shandong } \\
\text { (border } \\
\text { Jingjinji) }\end{array}$ & 1.90 \\
\hline Baoding(1) & Jingjinji & 1.93 & Changzhi(5) & $\begin{array}{c}\text { Shanxi } \\
\text { (border } \\
\text { J ingjinji) }\end{array}$ & 1.96 & Yilihasake(1) & Xinjiang & 1.85 \\
\hline
\end{tabular}


bioRxiv preprint doi: https://doi org/10.1101/2019.1220.884346; this version posted December 20, 2019. The copyright holder for this preprint (which was not certified by peer review) is the author/funder, who has granted bioRxiv a license to display the preprint in perpetuity. It is made available under aCC-BY 4.0 International license. the region where local economy was more developed such as Yangtze River Delta region, Pearl River Delta region and Sichuan Basin. There was a similar trend between

*(1),(2),(3),(4),(5),(6): the frequency of these cities ranking the top ten from 2014 to 2019

From Figure 5,it can be seen that northern cities or areas with higher concentration (including the highest mass concentration) of NO2 are also mostly situated in the Beijing-Tianjin-Hebei region and Xinjiang region from the year 2014 to 2017. Simultaneously, the concentration of NO2 took a high level position in Yangtze River Delta region, Pearl River Delta region and Sichuan Basin. It should be pointed that the distribution of $\mathrm{NO} 2$ was more extensive than other parameters, especially in the spatial variation of NO2 and it of the above PM2 5, PM10, AQI, CO and the

\begin{tabular}{|c|c|c|c|c|c|c|c|c|}
\hline \multicolumn{3}{|c|}{$2017 \mathrm{CO}$} & \multicolumn{3}{|c|}{$2018 \mathrm{CO}$} & \multicolumn{3}{|c|}{$2019 \mathrm{CO}$} \\
\hline City & $\begin{array}{c}\text { Belongs to } \\
\text { area }\end{array}$ & $\begin{array}{c}\text { Concentration } \\
/\left(\mu \mathrm{g} \cdot \mathrm{m}^{-3}\right)\end{array}$ & City & $\begin{array}{c}\text { Belongs to } \\
\text { area }\end{array}$ & $\begin{array}{c}\text { Concentration } \\
/\left(\mu \mathrm{g} \cdot \mathrm{m}^{-3}\right)\end{array}$ & City & $\begin{array}{c}\text { Belongs to } \\
\text { area }\end{array}$ & $\begin{array}{c}\text { Concentration } \\
/\left(\mu \mathrm{g} \cdot \mathrm{m}^{-3}\right)\end{array}$ \\
\hline Linfen(6) & $\begin{array}{c}\text { Shanxi } \\
\text { (border } \\
\text { Jingjinji) }\end{array}$ & 2.07 & Linfen(6) & $\begin{array}{c}\text { Shanxi } \\
\text { (border } \\
\text { J ingjinji) }\end{array}$ & 1.76 & Laiwu(1) & $\begin{array}{l}\text { Shandong } \\
\text { (border } \\
\text { Jingjinji) }\end{array}$ & 1.53 \\
\hline Jincheng(4) & $\begin{array}{c}\text { Shanxi } \\
\text { (border } \\
\text { Jingjinji) }\end{array}$ & 1.98 & Tangshan(6) & Jingjinji & 1.69 & Linfen(6) & $\begin{array}{c}\text { Shanxi } \\
\text { (border } \\
\text { Jingjinji) }\end{array}$ & 1.46 \\
\hline Tangshan(6) & Jingjinji & 1.96 & Panzhihua(2) & Sichuan & 1.53 & Tangshan(6) & J ingjinji & 1.37 \\
\hline Anyang(5) & $\begin{array}{c}\text { Henan } \\
\text { (border } \\
\text { jingjinji) }\end{array}$ & 1.85 & Anyang(5) & $\begin{array}{c}\text { Henan } \\
\text { (border } \\
\text { jingjinji) }\end{array}$ & 1.53 & Datong(2) & $\begin{array}{c}\text { Shanxi } \\
\text { (border } \\
\text { Jingjinji) }\end{array}$ & 1.35 \\
\hline Dekehasake(2) & Xinjiang & 1.83 & Dekehasake(2) & Xinjiang & 1.46 & Changzhi(5) & $\begin{array}{c}\text { Shanxi } \\
\text { (border } \\
\text { Jingjinji) }\end{array}$ & 1.25 \\
\hline Zibo(4) & $\begin{array}{r}\text { Shandong } \\
\text { (border } \\
\text { Jingjinji) }\end{array}$ & 1.76 & Loudi(1) & Hunan & 1.45 & Ganzhou(2) & Jiangxi & 1.24 \\
\hline Yuncheng(3) & $\begin{array}{c}\text { Shanxi } \\
\text { (border } \\
\text { Jingjinji) }\end{array}$ & 1.71 & Datong(2) & $\begin{array}{c}\begin{array}{c}\text { Shanxi } \\
\text { (border }\end{array} \\
\text { Jingjinji) }\end{array}$ & 1.42 & Jincheng $(4)$ & $\begin{array}{c}\text { Shanxi } \\
\text { (border } \\
\text { Jingjinji) }\end{array}$ & 1.23 \\
\hline Hebi(2) & $\begin{array}{c}\text { Henan } \\
\text { (border } \\
\text { jingjinji) }\end{array}$ & 1.68 & Ganzhou(2) & Jiangxi & 1.42 & Anyang(5) & $\begin{array}{c}\text { Henan } \\
\text { (border } \\
\text { jingjinji) }\end{array}$ & 1.21 \\
\hline Changzhi(5) & $\begin{array}{c}\text { Shanxi } \\
\text { (border } \\
\text { Jingjinji) }\end{array}$ & 1.67 & Xining(1) & Qinghai & 1.41 & Panzhihua(2) & Sichuan & 1.20 \\
\hline Xingtai & Jingjinji & 1.67 & Jincheng(4) & $\begin{array}{c}\text { Shanxi } \\
\text { (border } \\
\text { Jingjinji) }\end{array}$ & 1.38 & Penglai(2) & $\begin{array}{l}\text { Shandong } \\
\text { (border } \\
\text { Jingjinji) }\end{array}$ & 1.19 \\
\hline
\end{tabular}


following SO2, but it of the following $\mathrm{O} 3$ would indicate an opposite trend compared with CO. The highest mass concentration of NO2 was $56 \mu \mathrm{g} \cdot \mathrm{m}-3, \quad 59 \mu \mathrm{g} \cdot \mathrm{m}-3, \quad 60$
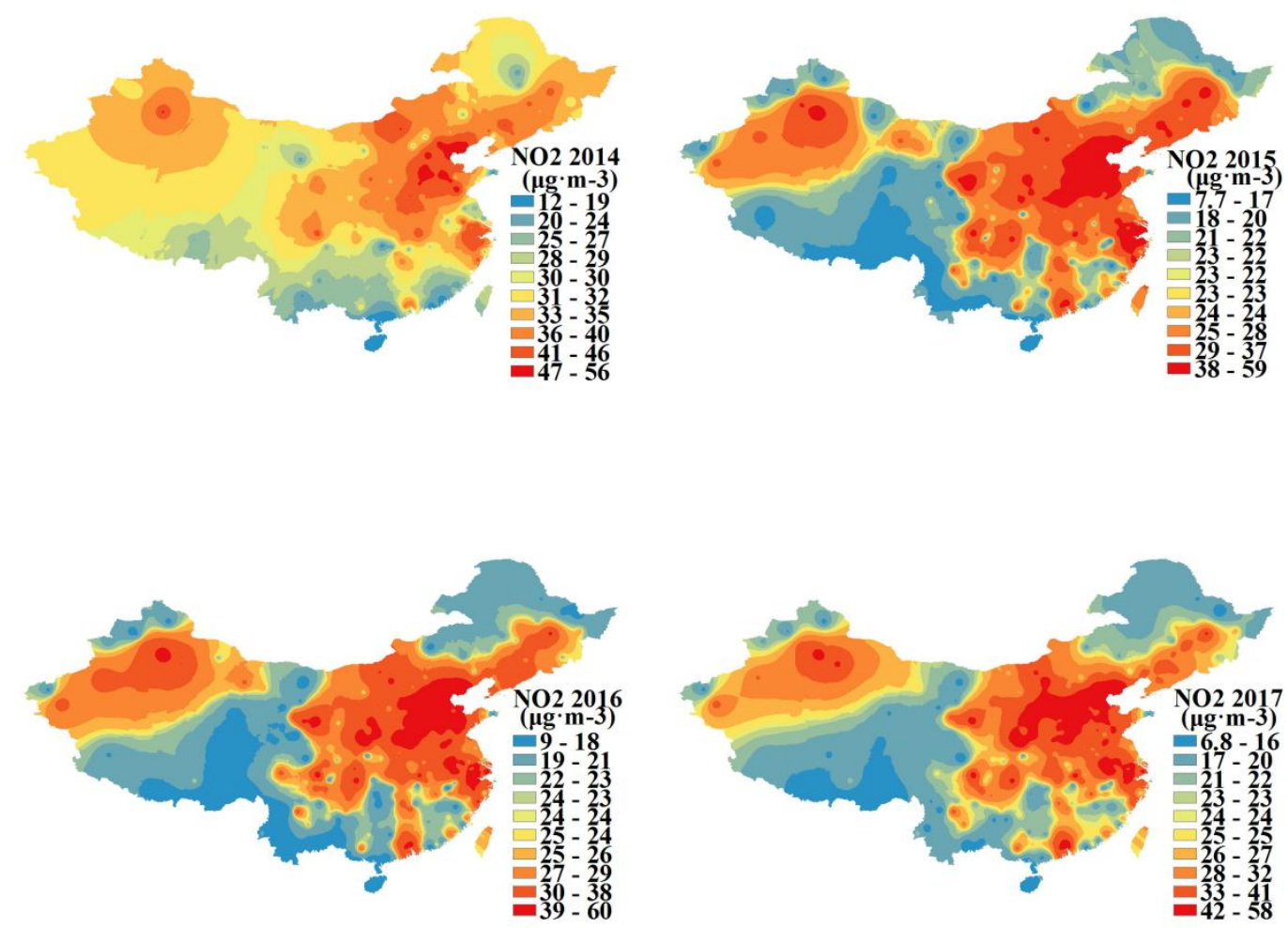

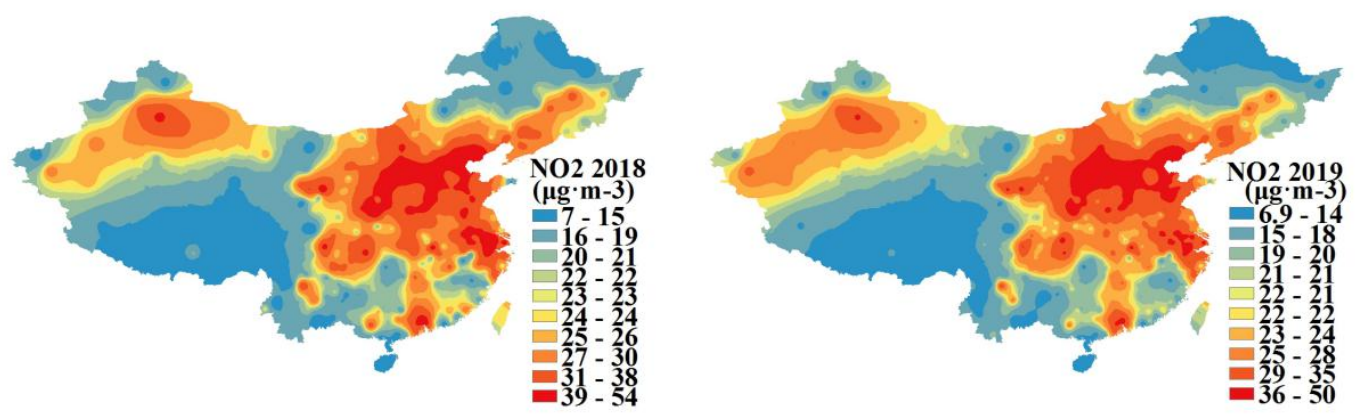

Figure 5. The spatial-temporal variation of the mass concentration of NO2 in accounted more than 2/3.Tangshan and Xingtai, two cities bordering.Beijing-Tianjintimes in these years. 
their affiliated regions from 2014 to 2019

\begin{tabular}{|c|c|c|c|c|c|c|c|c|}
\hline \multicolumn{3}{|c|}{$2014 \mathrm{NO} 2$} & \multicolumn{3}{|c|}{$2015 \mathrm{NO} 2$} & \multicolumn{3}{|c|}{$2016 \mathrm{NO} 2$} \\
\hline City & $\begin{array}{l}\text { Belongs to } \\
\text { area }\end{array}$ & $\begin{array}{c}\text { Concentration } \\
/\left(\mu \mathrm{g} \cdot \mathrm{m}^{-3}\right)\end{array}$ & City & $\begin{array}{l}\text { Belongs to } \\
\text { area }\end{array}$ & $\begin{array}{c}\text { Concentration } \\
/\left(\mu \mathrm{g} \cdot \mathrm{m}^{-3}\right)\end{array}$ & City & $\begin{array}{c}\text { Belongs to } \\
\text { area }\end{array}$ & $\begin{array}{c}\text { Concentration } \\
/\left(\mu \mathrm{g} \cdot \mathrm{m}^{-3}\right)\end{array}$ \\
\hline Zibo(3) & $\begin{array}{l}\text { Shandong } \\
\text { (border } \\
\text { jingjinji) }\end{array}$ & 65 & Zibo(3) & $\begin{array}{l}\text { Shandong } \\
\text { (border } \\
\text { jingjinji) }\end{array}$ & 61 & Xingtai(6) & Jingjinji & 61 \\
\hline Tangshan(6) & J ingjinji & 58 & Tangshan(6) & Jingjinji & 60 & Tangshan(6) & J ingjinji & 58 \\
\hline Linyi(1) & $\begin{array}{l}\text { Shandong } \\
\text { (border } \\
\text { jingjinji) }\end{array}$ & 57 & Xingtai(6) & J ingjinji & 59 & Baoding(3) & Jingjinji & 57 \\
\hline Xingtai(6) & J ingjinji & 56 & Zhengzhou(5) & $\begin{array}{c}\text { Henan } \\
\text { (border } \\
\text { jingjinji) }\end{array}$ & 55 & Handan(2) & J ingjinji & 54 \\
\hline Jinan(3) & $\begin{array}{l}\text { Shandong } \\
\text { (border } \\
\text { jingjinji) }\end{array}$ & 53 & Baoding(3) & J ingjinji & 53 & Huzhou(3) & Jiangsu & 54 \\
\hline Baoding(3) & J ingjinji & 52 & Linxiazhou(1) & Gansu & 53 & Shijiazhuang(2) & Jingjinji & 54 \\
\hline Beijing(1) & Jingjinji & 52 & Suzhou(2) & Jiangsu & 51 & Zhengzhou(5) & $\begin{array}{c}\text { Henan } \\
\text { (border } \\
\text { jingjinji) }\end{array}$ & 53 \\
\hline Suzhou(2) & Jiangsu & 51 & Jinan(3) & $\begin{array}{l}\text { Shandong } \\
\text { (border } \\
\text { jingjinji) }\end{array}$ & 51 & Zibo(3) & $\begin{array}{l}\text { Shandong } \\
\text { (border } \\
\text { jingjinji) }\end{array}$ & 52 \\
\hline Laiwu(2) & $\begin{array}{l}\text { Shandong } \\
\text { (border } \\
\text { jingjinji) }\end{array}$ & 51 & Zhuji(1) & Zhejiang & 51 & Hebi(1) & $\begin{array}{c}\text { Henan } \\
\text { (border } \\
\text { jingjinji) }\end{array}$ & 52 \\
\hline Qinhuangdao(2) & $\begin{array}{l}\text { Shandong } \\
\text { (border } \\
\text { jingjinji) }\end{array}$ & 50 & Xinxiang(2) & $\begin{array}{c}\text { Henan } \\
\text { (border } \\
\text { jingjinji) }\end{array}$ & 51 & Wulumuqi(1) & Xinjiang & 52 \\
\hline
\end{tabular}




\begin{tabular}{|c|c|c|c|c|c|c|c|c|}
\hline \multicolumn{3}{|c|}{2017 NO2 } & \multicolumn{3}{|c|}{2018 NO2 } & \multicolumn{3}{|c|}{2019 NO2 } \\
\hline City & $\begin{array}{c}\text { Belongs to } \\
\text { area }\end{array}$ & $\begin{array}{c}\text { Concentration } \\
/\left(\mu \mathrm{g} \cdot \mathrm{m}^{-3}\right)\end{array}$ & City & $\begin{array}{c}\text { Belongs to } \\
\text { area }\end{array}$ & $\begin{array}{c}\text { Concentration } \\
/\left(\mu \mathrm{g} \cdot \mathrm{m}^{-3}\right)\end{array}$ & City & $\begin{array}{c}\text { Belongs to } \\
\text { area }\end{array}$ & $\begin{array}{c}\text { Concentration } \\
/\left(\mu \mathrm{g} \cdot \mathrm{m}^{-3}\right)\end{array}$ \\
\hline Tangshan(6) & Jingjinji & 58 & Huzhou(3) & Jiangsu & 54 & Laiwu(2) & $\begin{array}{c}\text { Shandong } \\
\text { (border } \\
\text { jingjinji })\end{array}$ & 51 \\
\hline $\operatorname{Xian}(3)$ & Shan Xi & 58 & Tangshan(6) & Jingjinji & 54 & Tangshan(6) & Jingjinji & 49 \\
\hline Xingtai(6) & Jingjinji & 56 & $\operatorname{Xian}(3)$ & Shan Xi & 53 & Yunlin(1) & $\begin{array}{c}\text { Henan } \\
\text { (border } \\
\text { jingjinji) }\end{array}$ & 47 \\
\hline Huzhou(3) & Jiangsu & 55 & Changzhou(1) & Jiangsu & 49 & Xian(3) & Shan Xi & 46 \\
\hline Xianyang(2) & Shan Xi & 53 & Tianjin(2) & Jingjinji & 49 & Taiyuan(2) & $\begin{array}{c}\text { Shanxi } \\
\text { (border } \\
\text { jingjinji) }\end{array}$ & 44 \\
\hline Tianjin(2) & Jingjinji & 53 & Xingtai(6) & Jingjinji & 47 & Lvliang & $\begin{array}{c}\text { Shanxi } \\
\text { (border } \\
\text { jingjinji) }\end{array}$ & 44 \\
\hline Zhengzhou(5) & $\begin{array}{l}\text { Henan } \\
\text { (border } \\
\text { jingjinji) }\end{array}$ & 52 & Xinxiang(2) & $\begin{array}{l}\text { Henan } \\
\text { (border } \\
\text { jingjinji) }\end{array}$ & 47 & Huzhou(2) & Jiangsu & 44 \\
\hline Weinan(1) & Shan Xi & 51 & Xianyang(2) & Shan Xi & 47 & $\operatorname{Jinan}(3)$ & $\begin{array}{c}\begin{array}{c}\text { Shandong } \\
\text { (border } \\
\text { jingjinji })\end{array} \\
\text {. }\end{array}$ & 43 \\
\hline Handan(2) & Jingjinji & 51 & Taiyuan(2) & $\begin{array}{c}\text { Shanxi } \\
\text { (border } \\
\text { jingjinji) }\end{array}$ & 46 & Xingtai(6) & Jingjinji & 42 \\
\hline Shijiazhuang(2) & Jingjinji & 50 & Zhengzhou(5) & $\begin{array}{c}\text { Henan } \\
\text { (border } \\
\text { jingjinji) }\end{array}$ & 46 & Zhengzhou(5) & $\begin{array}{c}\text { Henan } \\
\text { (border } \\
\text { jingjinji) }\end{array}$ & 42 \\
\hline
\end{tabular}

Hebei region on the right side from the year 2014 to 2019. In fact, the spatial variation 

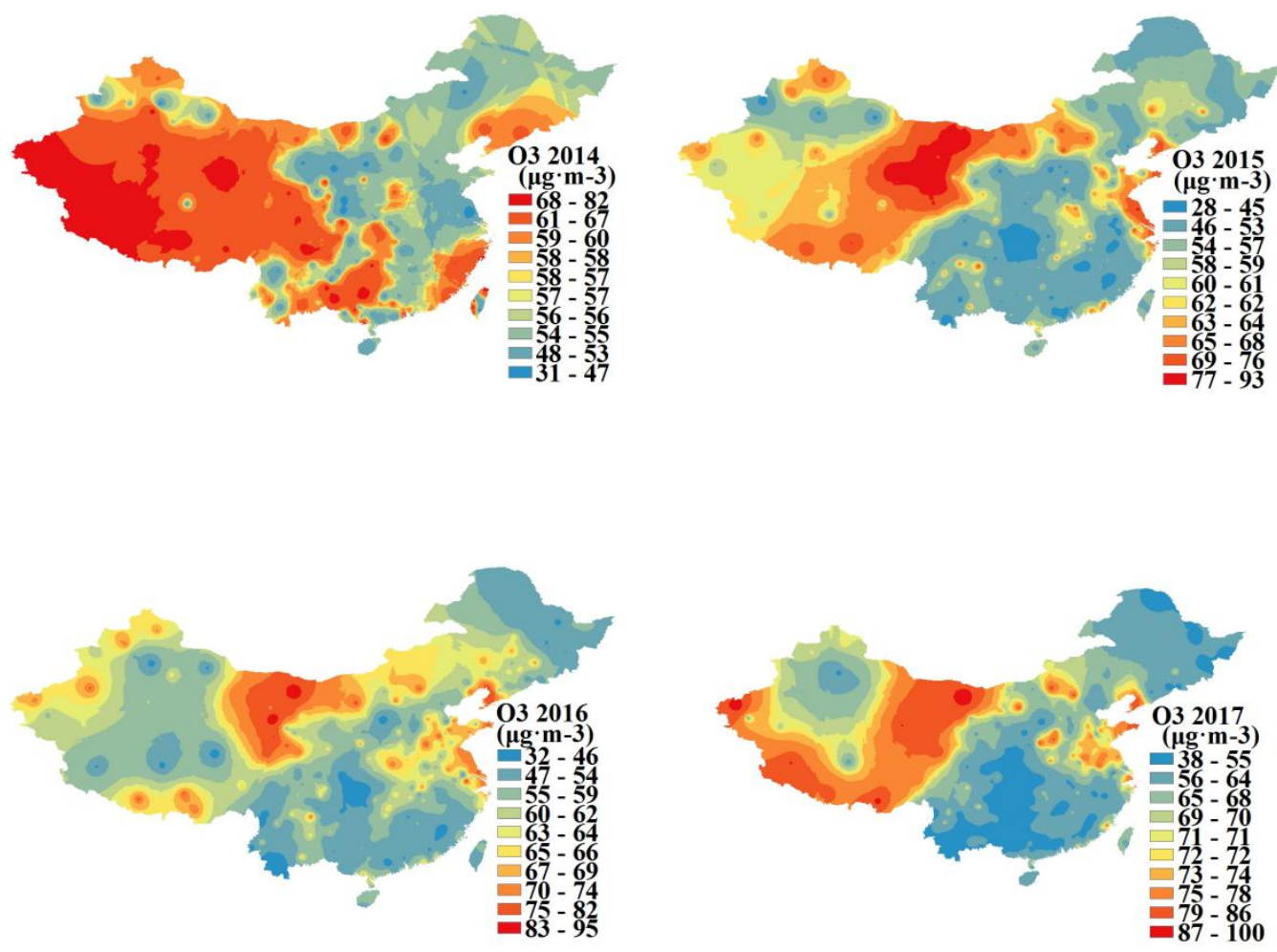

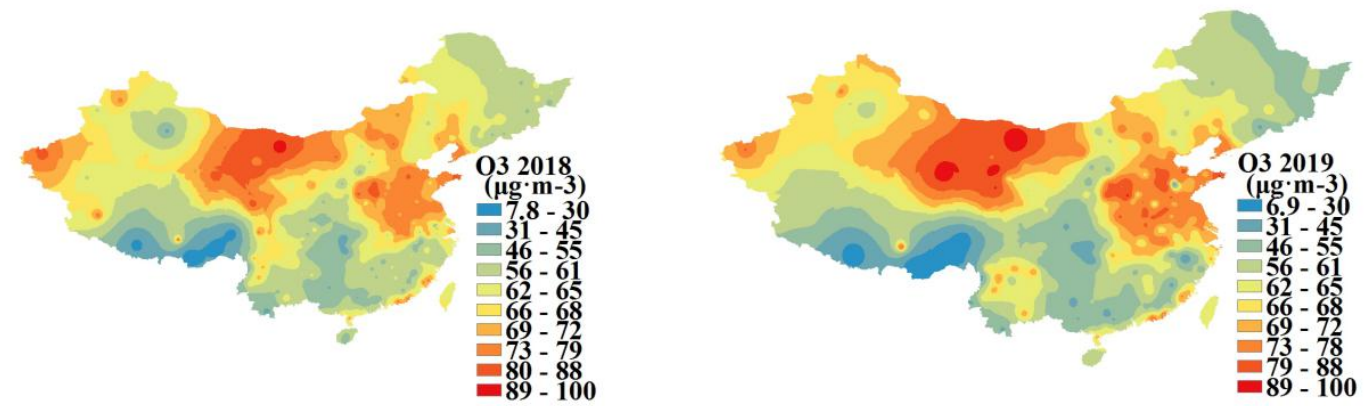

Figure 6. The spatial-temporal variation of the mass concentration of $\mathrm{O3}$ in mainland China from 2014 to 2019 


\begin{tabular}{|c|c|c|c|c|c|c|c|c|}
\hline \multicolumn{3}{|c|}{$2014 \mathrm{O} 3$} & \multicolumn{3}{|c|}{$2015 \mathrm{O} 3$} & \multicolumn{3}{|c|}{$2016 \mathrm{O} 3$} \\
\hline City & $\begin{array}{l}\text { Belongs to } \\
\text { area }\end{array}$ & $\begin{array}{c}\text { Concentration/ } \\
\left(\mu \mathrm{g} \cdot \mathrm{m}^{-3}\right)\end{array}$ & City & $\begin{array}{c}\text { Belongs to } \\
\text { area }\end{array}$ & $\begin{array}{c}\text { Concentration/ } \\
\left(\mu \mathrm{g} \cdot \mathrm{m}^{-3}\right)\end{array}$ & City & $\begin{array}{l}\text { Belongs to } \\
\text { area }\end{array}$ & $\begin{array}{c}\text { Concentration } \\
/\left(\mu \mathrm{g} \cdot \mathrm{m}^{-3}\right)\end{array}$ \\
\hline Rongcheng(3) & $\begin{array}{l}\text { Shandong } \\
\text { (border } \\
\text { jingjinji) }\end{array}$ & 93 & Haixizhou(3) & Qinghai & 97 & Haibeizhou(4) & Qinghai & 95 \\
\hline Weihai(4) & $\begin{array}{l}\text { Shandong } \\
\text { (border } \\
\text { jingjinji) }\end{array}$ & 92 & Alashanmeng(4) & Neimenggu & 94 & Alashanmeng(4) & Neimenggu & 95 \\
\hline Penglai(1) & $\begin{array}{l}\text { Shandong } \\
\text { (border } \\
\text { jingjinji) }\end{array}$ & 87 & Hainanzhou(3) & Qinghai & 94 & Hainanzhou(3) & Qinghai & 83 \\
\hline $\operatorname{Linan}(1)$ & Zhejiang & 85 & Haibeizhou(4) & Qinghai & 90 & Yixing(1) & J iangsu & 82 \\
\hline Jiaonan(2) & $\begin{array}{l}\text { Shandong } \\
\text { (border } \\
\text { jingjinji) }\end{array}$ & 82 & Yanzheng(1) & Jiangsu & 82 & Guoluozhou(3) & Qinghai & 81 \\
\hline Weifang(1) & $\begin{array}{l}\text { Shandong } \\
\text { (border } \\
\text { jingjinji) }\end{array}$ & 81 & Jiayuguan(2) & Gansu & 81 & Yingkou(2) & Liaoning & 81 \\
\hline Wendeng(1) & $\begin{array}{l}\text { Shandong } \\
\text { (border } \\
\text { jingjinji) }\end{array}$ & 81 & Guoluozhou(3) & Qinghai & 81 & Jiayuguan(2) & Gansu & 81 \\
\hline Dongying(1) & $\begin{array}{l}\text { Shandong } \\
\text { (border } \\
\text { jingjinji) }\end{array}$ & 80 & Yingkou(2) & Liaoning & 80 & Eerduosi(2) & Neimenggu & 80 \\
\hline Eerduosi(2) & Neimenggu & 80 & Lasa(1) & Xizang & 80 & Jiaonan(1) & Jiangsu & 80 \\
\hline Qingdao(1) & $\begin{array}{l}\text { Shandong } \\
\text { (border } \\
\text { jingjinji) }\end{array}$ & 80 & Weihai(4) & $\begin{array}{l}\text { Shandong } \\
\quad \text { (border } \\
\text { jingjinji) }\end{array}$ & 79 & Dalian(1) & Liaoning & 79 \\
\hline
\end{tabular}


bioRxiv preprint doi: https://doi org/10.1101/2019.1220.884346; this version posted December 20, 2019. The copyright holder for this preprint (which was not certified by peer review) is the author/funder, who has granted bioRxiv a license to display the preprint in perpetuity. It is made available under aCC-BY 4.0 International license.

\begin{tabular}{|c|c|c|c|c|c|c|c|c|}
\hline \multicolumn{3}{|c|}{$2017 \mathrm{NO} 2$} & \multicolumn{3}{|c|}{$2018 \mathrm{NO} 2$} & \multicolumn{3}{|c|}{$2019 \mathrm{NO} 2$} \\
\hline City & $\begin{array}{l}\text { Belongs to } \\
\text { area }\end{array}$ & $\begin{array}{c}\text { Concentration/ } \\
\left(\mu \mathrm{g} \cdot \mathrm{m}^{-3}\right)\end{array}$ & City & $\begin{array}{l}\text { Belongs to } \\
\text { area }\end{array}$ & $\begin{array}{c}\text { Concentration/ } \\
\left(\mu \mathrm{g} \cdot \mathrm{m}^{-3}\right)\end{array}$ & City & $\begin{array}{l}\text { Belongs to } \\
\text { area }\end{array}$ & $\begin{array}{c}\text { Concentration } \\
/\left(\mu \mathrm{g} \cdot \mathrm{m}^{-3}\right)\end{array}$ \\
\hline Enshizhou(1) & Hubei & 37 & Alashanmeng(4) & Neimenggu & 105 & Haixizhou(3) & Qinghai & 104 \\
\hline Xiangxi & Hunan & 38 & Guoluozhou(3) & Qinghai & 90 & Alashanmeng(4) & Neimenggu & 102 \\
\hline Qiandongnanzhou(1) & Guizhou & 38 & Kezhou(1) & Xinjiang & 89 & Haibeizhou(4) & Qinghai & 96 \\
\hline Xishuangbanna(1) & Yunnan & 40 & Weihai(4) & $\begin{array}{l}\text { Shandong } \\
\text { (border } \\
\text { jingjinji) }\end{array}$ & 89 & Weihai(4) & $\begin{array}{l}\text { Shandong } \\
\text { (border } \\
\text { jingjinji) }\end{array}$ & 92 \\
\hline Qianxinanzhou(1) & Guizhou & 40 & Haixizhou(3) & Qinghai & 88 & Hainanzhou(3) & Qinghai & 89 \\
\hline Nujiang(1) & Yunnan & 41 & Jiaozuo(2) & $\begin{array}{c}\text { Henan } \\
\text { (border } \\
\text { jingjinji) }\end{array}$ & 87 & Jiaozuo(2) & $\begin{array}{c}\text { Henan } \\
\text { (border } \\
\text { jingjinji) }\end{array}$ & 89 \\
\hline Chongqing(1) & Chongqing & 42 & Haibeizhou(4) & Qinghai & 87 & Rongcheng(3) & $\begin{array}{l}\text { Shandong } \\
\text { (border } \\
\text { jingjinji) }\end{array}$ & 89 \\
\hline Wuzhou(1) & Guangxi & 42 & Rongcheng(3) & $\begin{array}{l}\text { Shandong } \\
\text { (border } \\
\text { jingjinji) }\end{array}$ & 85 & J inchang(1) & Gansu & 86 \\
\hline Hechi(1) & Jiangsu & 43 & Qingyang(1) & Gansu & 85 & Changzhi(2) & $\begin{array}{c}\text { Shanxi } \\
\text { (border } \\
\text { jingjinji) }\end{array}$ & 85 \\
\hline Pingxiang(1) & Jiangxi & 43 & Changzhi(2) & $\begin{array}{c}\text { Shanxi } \\
\text { (border } \\
\text { jingjinji) }\end{array}$ & 84 & Jincheng(1) & $\begin{array}{c}\text { Shanxi } \\
\text { (border } \\
\text { jingjinji) }\end{array}$ & 84 \\
\hline
\end{tabular}

*(1),(2),(3),(4),(5),(6): the frequency of these cities ranking the top ten from 2014 to 2019

From Figure 7, it can be seen that the area with higher concentration (including the highest mass concentration) of SO2 was mainly situated in Shanxi and Shandong provinces nearby Beijing-Tianjin-Hebei region and a small part of areas bordering such places (e.g. Liaoning province and Inner Mongolia region) from the year 2014 to 2019. There was a similar trend between the spatial variation of SO2 and that of the above PM2.5, PM10, AQI, CO and NO2, but a different trend between the spatial variation of $\mathrm{SO} 2$ and that of the above O3. The highest mass concentration of SO2 was $83 \mu \mathrm{g} \cdot \mathrm{m}-3$, $76 \mu \mathrm{g} \cdot \mathrm{m}-3, \quad 87 \mu \mathrm{g} \cdot \mathrm{m}-3,82 \mu \mathrm{g} \cdot \mathrm{m}-3, \quad 48 \mu \mathrm{g} \cdot \mathrm{m}-3,37 \mu \mathrm{g} \cdot \mathrm{m}-3$ in each separated year from 2014 to 2019. Among these, the highest concentration of SO2 was $87 \mu \mathrm{g} \cdot \mathrm{m}-3$ in 2016 , which was higher than the other five years and more than two times as high as that in 
bioRxiv preprint doi: https://doi org/10.1101/2019.12 20 884346; this version posted December 20, 2019. The copyright holder for this

preprint (which was not certified by peer review) is the author/funder, who has granted bioRxiv a license to display the preprint in perpetuity. It is made available under aCC-BY 4.0 International license.
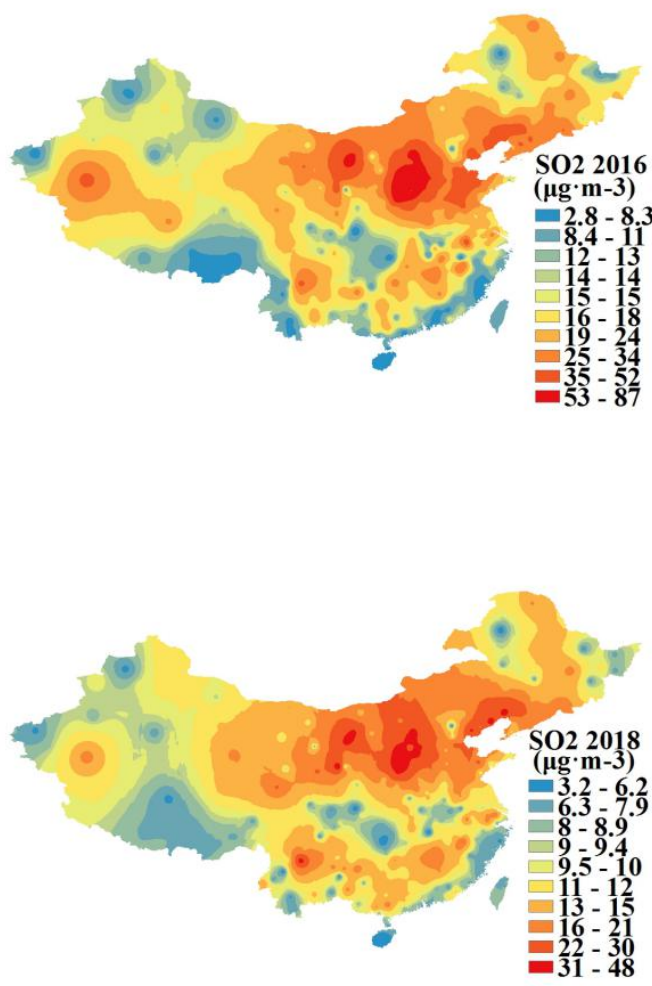
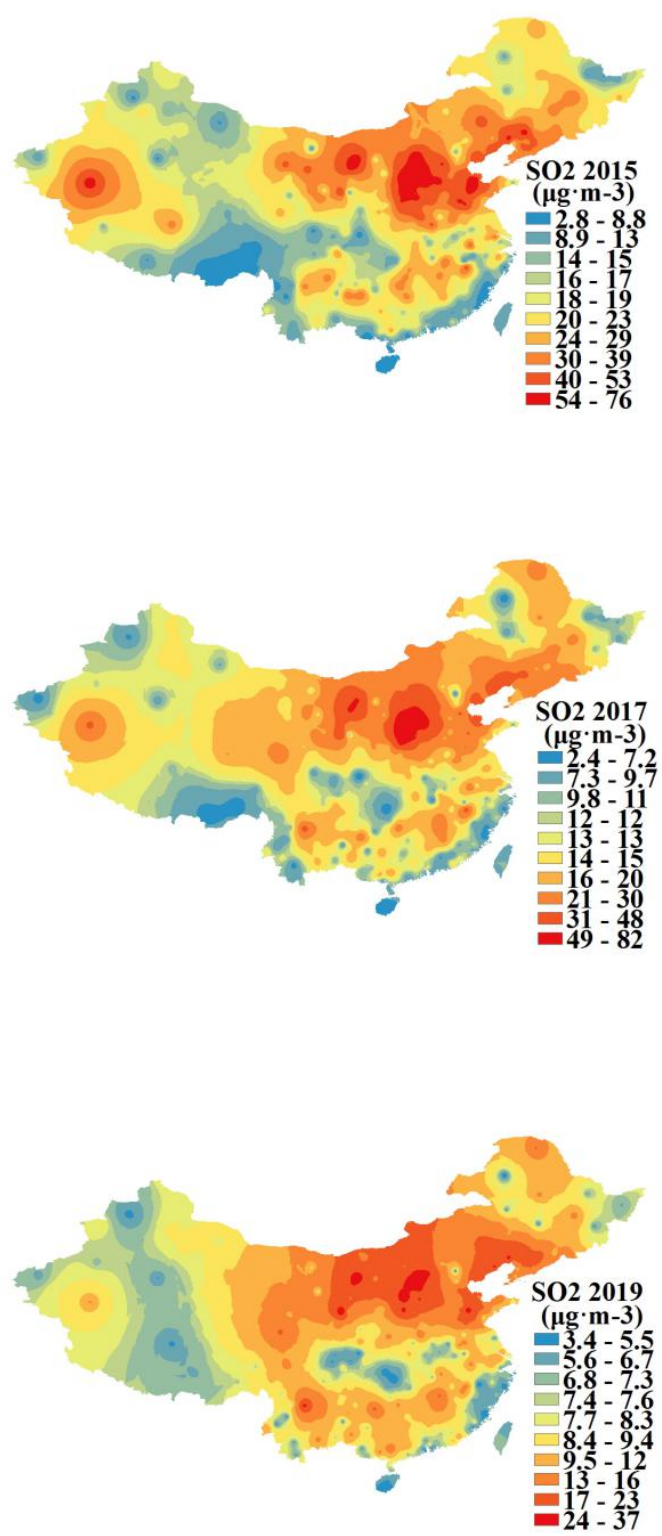

region, with 1/6 in Shandong province on the right side of Beijing-Tianjin-Hebei region, 
bioRxiv preprint doi: https://doi.org/10.1101/2019.12.20.884346; this version posted December 20, 2019. The copyright holder for this preprint (which was not certified by peer review) is the author/funder, who has granted bioRxiv a license to display the preprint in perpetuity. It is made available under aCC-BY 4.0 International license.

356

357

358

359

360

361

362

363

364

365

366

367

368

369

370

371 
their affiliated regions from 2014 to 2019.

\begin{tabular}{|c|c|c|c|c|c|c|c|c|}
\hline \multicolumn{3}{|c|}{$2014 \mathrm{SO} 2$} & \multicolumn{3}{|c|}{$2015 \mathrm{SO} 2$} & \multicolumn{3}{|c|}{$2016 \mathrm{SO} 2$} \\
\hline City & Belongs to area & $\begin{array}{c}\text { Concentration } \\
/\left(\mu \mathrm{g} \cdot \mathrm{m}^{-3}\right)\end{array}$ & City & Belongs to area & $\begin{array}{c}\text { Concentration } \\
/\left(\mu \mathrm{g} \cdot \mathrm{m}^{-3}\right)\end{array}$ & City & $\begin{array}{l}\text { Belongs to } \\
\text { area }\end{array}$ & $\begin{array}{c}\text { Concentration } \\
/\left(\mu \mathrm{g} \cdot \mathrm{m}^{-3}\right)\end{array}$ \\
\hline Zibo(2) & $\begin{array}{c}\text { Shandong } \\
\text { (border } \\
\text { jingjinji) }\end{array}$ & 108 & Zibo(2) & $\begin{array}{c}\text { Shandong } \\
\text { (border } \\
\text { jingjinji) }\end{array}$ & 82 & Jinzhong(4) & $\begin{array}{l}\text { Shanxi } \\
\text { (border } \\
\text { jingjinji) }\end{array}$ & 87 \\
\hline Yangquan(3) & $\begin{array}{l}\text { Shanxi } \\
\text { (border } \\
\text { jingjinji) }\end{array}$ & 85 & Shuozhou(3) & $\begin{array}{c}\text { Shanxi } \\
\text { (border } \\
\text { jingjinji) }\end{array}$ & 76 & Linfen(6) & $\begin{array}{c}\text { Shanxi } \\
\text { (border } \\
\text { jingjinji) }\end{array}$ & 82 \\
\hline Laiwu(2) & $\begin{array}{c}\text { Shandong } \\
\text { (border } \\
\text { jingjinji) }\end{array}$ & 81 & Hetiandiqu(1) & Xinjiang & 72 & Shuozhou(3) & $\begin{array}{l}\text { Shanxi } \\
\text { (border } \\
\text { jingjinji) }\end{array}$ & 67 \\
\hline Taiyuan(4) & $\begin{array}{l}\text { Shanxi } \\
\text { (border } \\
\text { jingjinji) }\end{array}$ & 66 & Jinzhong(4) & $\begin{array}{c}\text { Shanxi } \\
\text { (border } \\
\text { jingjinji) }\end{array}$ & 72 & Shijiazui(5) & Ningxia & 67 \\
\hline Jining & $\begin{array}{l}\text { Shandong } \\
\text { (border } \\
\text { jingjinji) }\end{array}$ & 64 & Shijiazui(5) & Ningxia & 71 & Jincheng $(1)$ & $\begin{array}{l}\text { Shanxi } \\
\text { (border } \\
\text { jingjinji) }\end{array}$ & 66 \\
\hline Shandong & $\begin{array}{c}\text { Shandong } \\
\text { (border } \\
\text { jingjinji) }\end{array}$ & 64 & Taiyuan(4) & $\begin{array}{l}\text { Shanxi } \\
\text { (border } \\
\text { jingjinji) }\end{array}$ & 68 & Yuncheng(2) & $\begin{array}{l}\text { Shanxi } \\
\text { (border } \\
\text { jingjinji) }\end{array}$ & 66 \\
\hline Zaozhuang & $\begin{array}{c}\text { Shandong } \\
\text { (border } \\
\text { jingjinji) }\end{array}$ & 63 & Lvliang(5) & $\begin{array}{c}\text { Shanxi } \\
\text { (border } \\
\text { jingjinji) }\end{array}$ & 68 & Taiyuan(4) & $\begin{array}{c}\text { Shanxi } \\
\text { (border } \\
\text { jingjinji) }\end{array}$ & 65 \\
\hline $\operatorname{Linfen}(6)$ & $\begin{array}{l}\text { Shanxi } \\
\text { (border } \\
\text { jingjinji) }\end{array}$ & 63 & Zhangqiu(1) & $\begin{array}{c}\text { Shandong } \\
\text { (border } \\
\text { jingjinji) }\end{array}$ & 64 & Lvliang(5) & $\begin{array}{l}\text { Shanxi } \\
\text { (border } \\
\text { jingjinji) }\end{array}$ & 62 \\
\hline Shijiazui(5) & Ningxia & 59 & Linfen(6) & $\begin{array}{l}\text { Shanxi } \\
\text { (border } \\
\text { jingjinji) }\end{array}$ & 63 & Yangquan(3) & $\begin{array}{l}\begin{array}{l}\text { Shanxi } \\
\text { (border }\end{array} \\
\text { jingjinji) }\end{array}$ & 61 \\
\hline Shenyang(1) & $\begin{array}{c}\text { Liaoning } \\
\text { (border } \\
\text { jingjinji) }\end{array}$ & 57 & Wuhai(4) & Neimenggu & 62 & Changzhi(1) & $\begin{array}{l}\text { Shanxi } \\
\text { (border } \\
\text { jingjinji) }\end{array}$ & 61 \\
\hline
\end{tabular}




\begin{tabular}{|c|c|c|c|c|c|c|c|c|}
\hline \multicolumn{3}{|c|}{$2017 \mathrm{SO} 2$} & \multicolumn{3}{|c|}{$2018 \mathrm{SO} 2$} & \multicolumn{3}{|c|}{2019 SO2 } \\
\hline City & Belongs to area & $\begin{array}{c}\text { Concentration } \\
/\left(\mu \mathrm{g} \cdot \mathrm{m}^{-3}\right)\end{array}$ & City & Belongs to area & $\begin{array}{c}\text { Concentration } \\
/\left(\mu \mathrm{g} \cdot \mathrm{m}^{-3}\right)\end{array}$ & City & $\begin{array}{l}\text { Belongs to } \\
\text { area }\end{array}$ & $\begin{array}{c}\text { Concentration } \\
/\left(\mu \mathrm{g} \cdot \mathrm{m}^{-3}\right)\end{array}$ \\
\hline Jinzhong(4) & $\begin{array}{c}\text { Shanxi } \\
\text { (border } \\
\text { jingjinji) }\end{array}$ & 83 & Linfen(6) & $\begin{array}{c}\text { Shanxi } \\
\text { (border } \\
\text { jingjinji) }\end{array}$ & 48 & Baiyin(2) & Ningxia & 39 \\
\hline Linfen(6) & $\begin{array}{c}\text { Shanxi } \\
\text { (border } \\
\text { jingjinji) }\end{array}$ & 79 & Baiyin(2) & Ningxia & 42 & Wuhai(4) & Neimenggu & 33 \\
\hline Lvliang(5) & $\begin{array}{c}\text { Shanxi } \\
\text { (border } \\
\text { jingjinji) }\end{array}$ & 68 & Lvliang(5) & $\begin{array}{c}\text { Shanxi } \\
\text { (border } \\
\text { jingjinji) }\end{array}$ & 39 & Laiwu(2) & $\begin{array}{c}\text { Shandong } \\
\text { (border } \\
\text { jingjinji) }\end{array}$ & 32 \\
\hline Shijiazui(5) & Ningxia & 51 & Panzhihua(2) & Sichuan & 38 & Shuozhou(3) & $\begin{array}{c}\text { Shanxi } \\
\text { (border } \\
\text { jingjinji) }\end{array}$ & 29 \\
\hline Taiyuan(4) & $\begin{array}{c}\text { Shanxi } \\
\text { (border } \\
\text { jingjinji) }\end{array}$ & 51 & Jinzhou(2) & $\begin{array}{l}\text { Liaoning } \\
\text { (border } \\
\text { jingjinji) }\end{array}$ & 37 & Panzhihua(2) & Sichuan & 29 \\
\hline Yinchuang(2) & Ningxia & 51 & Shijiazui(5) & Ningxia & 37 & Xinzhou(2) & $\begin{array}{c}\text { Shanxi } \\
\text { (border } \\
\text { jingjinji) }\end{array}$ & 29 \\
\hline Yuncheng(2) & $\begin{array}{c}\text { Shanxi } \\
\text { (border } \\
\text { jingjinji) }\end{array}$ & 51 & Jinzhong(4) & $\begin{array}{c}\text { Shanxi } \\
\text { (border } \\
\text { jingjinji) }\end{array}$ & 36 & Lvliang(5) & $\begin{array}{c}\text { Shanxi } \\
\text { (border } \\
\text { jingjinji) }\end{array}$ & 29 \\
\hline Wuhai(4) & Neimenggu & 51 & Yinchuang(2) & Ningxia & 36 & Datong(1) & $\begin{array}{c}\text { Shanxi } \\
\text { (border } \\
\text { jingjinji) }\end{array}$ & 28 \\
\hline Yangquan(3) & $\begin{array}{c}\text { Shanxi } \\
\text { (border } \\
\text { jingjinji) }\end{array}$ & 49 & Huludao(1) & $\begin{array}{r}\text { Liaoning } \\
\text { (border } \\
\text { jingjinji) }\end{array}$ & 36 & Linfen(6) & $\begin{array}{l}\text { Shanxi } \\
\text { (border } \\
\text { jingjinji) }\end{array}$ & 27 \\
\hline Xinzhou(2) & $\begin{array}{c}\text { Shanxi } \\
\text { (border } \\
\text { jingjinji) }\end{array}$ & 48 & Wuhai(4) & Neimenggu & 34 & J inzhou(2) & $\begin{array}{l}\text { Liaoning } \\
\text { (border } \\
\text { jingjinji) }\end{array}$ & 27 \\
\hline
\end{tabular}

\section{Conclusion}


Henan provinces and Xinjiang areas in the west of mainland China. In addition, the concentration of $\mathrm{NO} 2$ was also higher in such economically developed regions as Yangtze River Delta, Pearl River Delta and Sichuan Basin, which indicates the distribution of NO2 was of universality, a different characteristic compared with the concentricity occupied by the other parameters. By contrast, $\mathrm{O} 3$, one of the atmospheric pollution parameters, was mainly distributed in Qinghai and Inner Mongolia in the central north of mainland China. PM2.5, AQI and PM10 experienced almost the same variation trend with time, which also happened to $\mathrm{CO}, \mathrm{NO} 2$ and $\mathrm{SO} 2$. Whereas, $\mathrm{O} 3$ witnessed an opposite variation trend to $\mathrm{CO}, \mathrm{NO} 2$ and $\mathrm{SO} 2$ with time. These cities including Baoding, Xingtai, Handan, Tangshan in Beijing-Tianjin-Hebei region, Langfang in Shandong province, Linfen in Shanxi province, haibeizhou in Qinghai, Kashi, Hetian, Aksu and Kezhou in Xinjiang should be emphatically improved. Except for the decline of the highest concentration of CO and SO2 from 2014 to 2019, this indicator of other parameters was not improved. In general, the atmospheric environment of mainland China had not been obviously improved over the six years. As a consequence, the study in this paper urges us to pay attention to the problem with the atmospheric environment of northern region of China, and also indicates cities and regions with severer atmospheric contamination, for which authentic actions should be implemented to address the corresponding problems.

\section{References}


(3) :592 597. university 2012.

421 ultrafine particles and their effects on health [J]. Environmental and Occupational

422 Medicine, 2007, 24(1): 80-83. 
Science, 2009, 29(3): 482-487.

[9] Xie Peng, Liu Xiaoyun, Liu Zhaorong, et al. Study on the relationship between exposure and atmospheric pollution of particulate matter in China[J]. China Environmental Science, 2009, 29(10): 1034-1040.

[10] Duan Yusen, Zhang Yuhua, Wang Dongfang, et al. Analysis of temporal and spatial distribution characteristics of ozone pollution in some cities ofChina $[\mathrm{J}]$. Environmental Monitoring Managementand Technology, 2011(S1): 34-39.

[11] Yang Junyi, Xin Jinyuan, Ji Dongsheng, et al. Analysis of changes in air pollution in the background of the Beijing-Tianjin-Hebei region in the summer of 20082011. Environmental Science, 2012, 33(11): 3693-3704.

[12] WANG Ying,LI Lingjun,LIU Yang.Characteristics of atmospheric NO2 pollution in Beijing-Tianjin-Hebei region and the Yangtze River Delta region[J].Environmental science,2012, 33(11): 3685-3692.

[13] Wang Xiquan, Yang Ting, Wang Zifa.Analysis of the Impact of CrossControl Area of AshPollution on the Case of Beijing-Tianjin-Hebei and Northeast China Ash Pollution[J].Climatic and Environmental Research,2011, 16(6): 690-696.

[14] LIU Xiaoyun, XIE Peng, LIU Zhaorong, et al.Economic loss assessment of acute health effects of particulate matter pollution in the Pearl River Delta[J]. Journal of Peking University(Natural Science), 2010, 46(5): 829-834.

[15] Liu Jie, Yang,Peng,Lv, Wensheng,Pollution characteristics of particulate 
445

446

447

448

449

450

451

452

453

454

455

456

457

458

459

460

461

462

463

464

and interpolation analysis of its spatial distribution in the Beijing are[J]a.Journal of University of Scienceand Technology Beijing,2014,36(9):1269-1279.

[16] Liu Jie,Yang,Peng,Lv,Wensheng,LIU Agudamu,LIU Junxiu,Study on the variation of mass concentration of atmospheric particles in the 6th district of Beijing City Journal of Safety and Environmen,2015,15(6):333-339.

[17] Kong S F, Shi J W, Lu B, et al. Characterization of PAHs within PM1O fractionfor ashes from coke production, iron smelt, heating station and power plantstacks in Liaoning Province, China [J]. Atmospheric Environment, 2011, 45 (23):3777-3785.

[18] Shaofei Kong, Yonghai Xu, YongBing Lu, Zhipeng Bai, Xueyan Zhao, Li

Chen, Bin Han, Zhiyong Li, Yaqin Ji Liu, Hua Jiang. Potential threat of heavy metals in resuspended dusts onbuilding surfaces in oilfield city. Atmospheric Environment. 2011, 45: 4192-4204 (SCI, 2011IF=3.226);

[19] Kong shaofeiStudy on The Chemical Composition, Risk Assessment and Emission Inventory Establishment for Hazardous Components in Particulate Matter from Atmospheric Pollution Sources[D], Tianjin,Nankaidaxue,2012

[20] Liu Jie Temporal - spatial Variation as well as Evaluation and Prediction Models of Air pollutants in Beijing[D],beijing,Beijingkelidaxue,2015

[21] Beijing Municipal Statistics Bureau,http://tjj.beijing.gov.cn/tjsj/

[22] ZHAO Wen-xia, YANG Zhao-xu, LIU Shuai, et al. Composition and 
465 combustion dynamics analysis of typical crop straws[J]. Journal of Agro-Environment

466 Science, 2019,38 (4) : 921-927.

467 

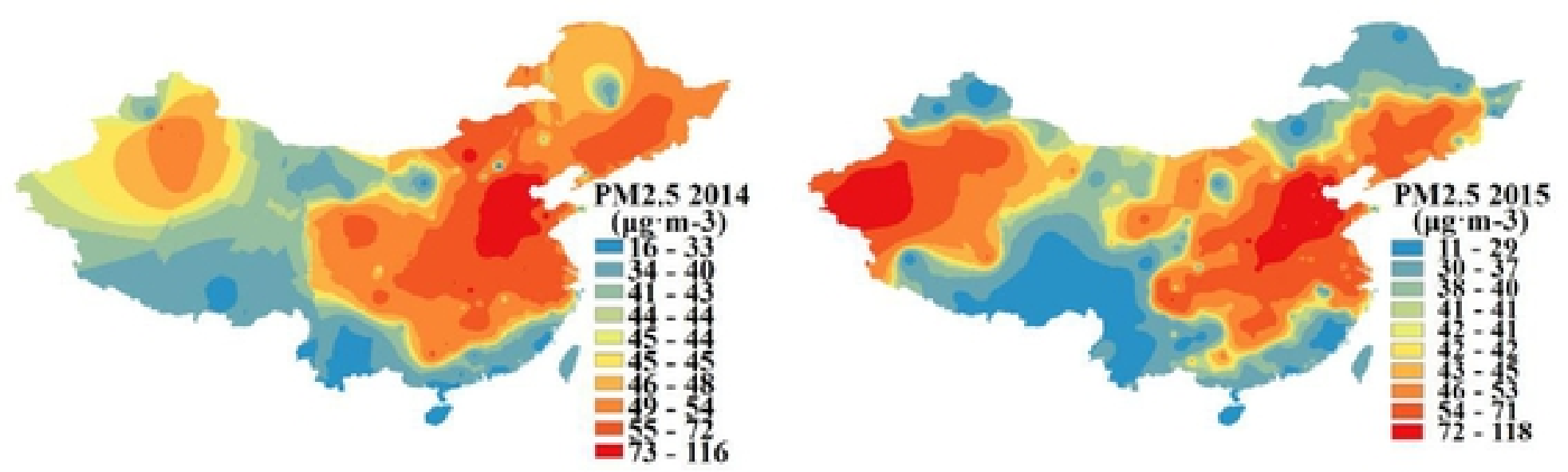
bioRxiv preprint doi: https://doi.org/10.1101/2019.12.20.884346; this version posted December 20, 2019. The copyright holder for this
preprint (which was not certified by peer review) is the author/funder, who has granted bioRxiv a license to display the preprint in
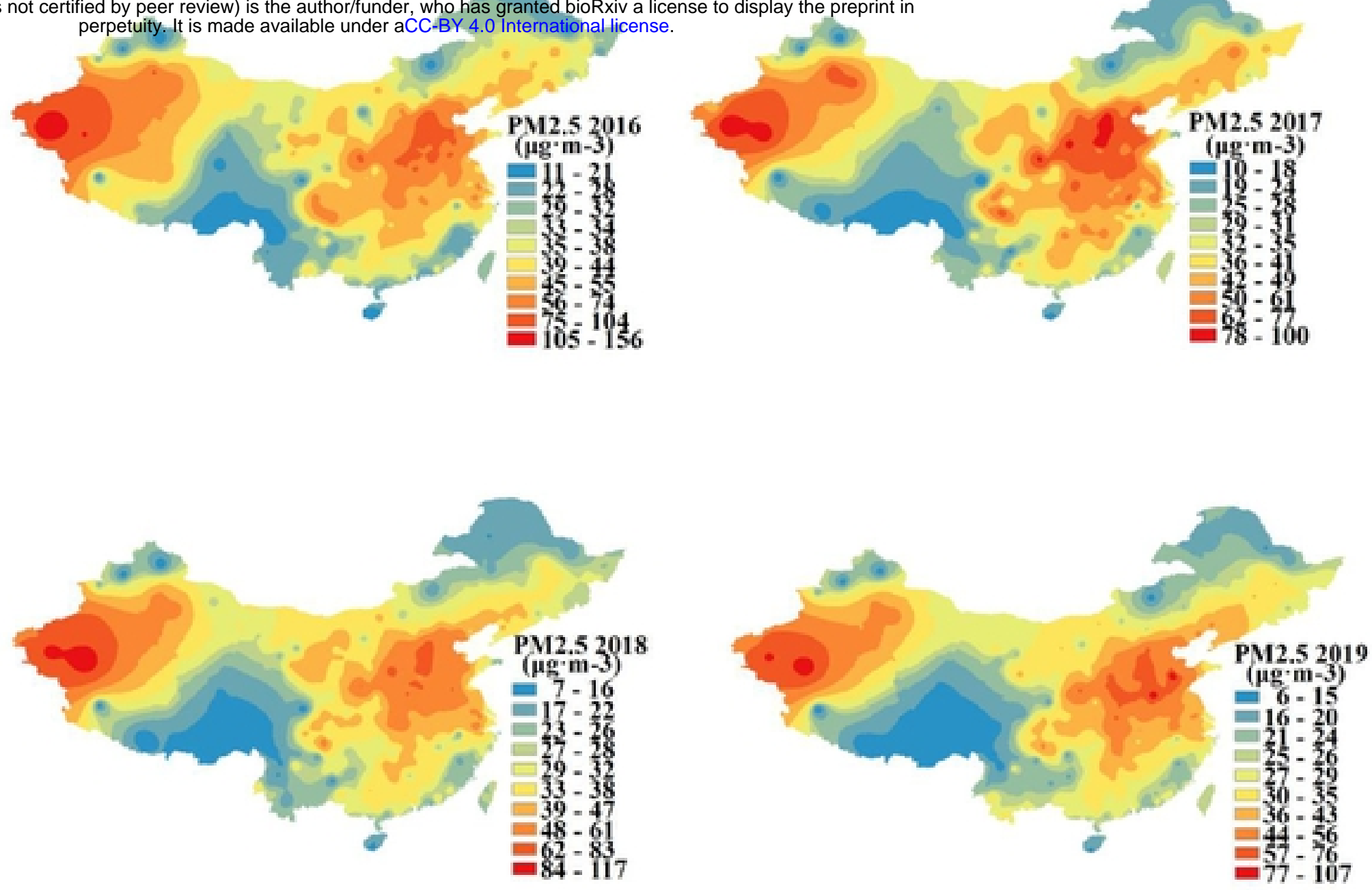

Figure 1. The spatial-temporal variation of the mass concentration of PM2.5 in mainland China from 2014 to 2019.
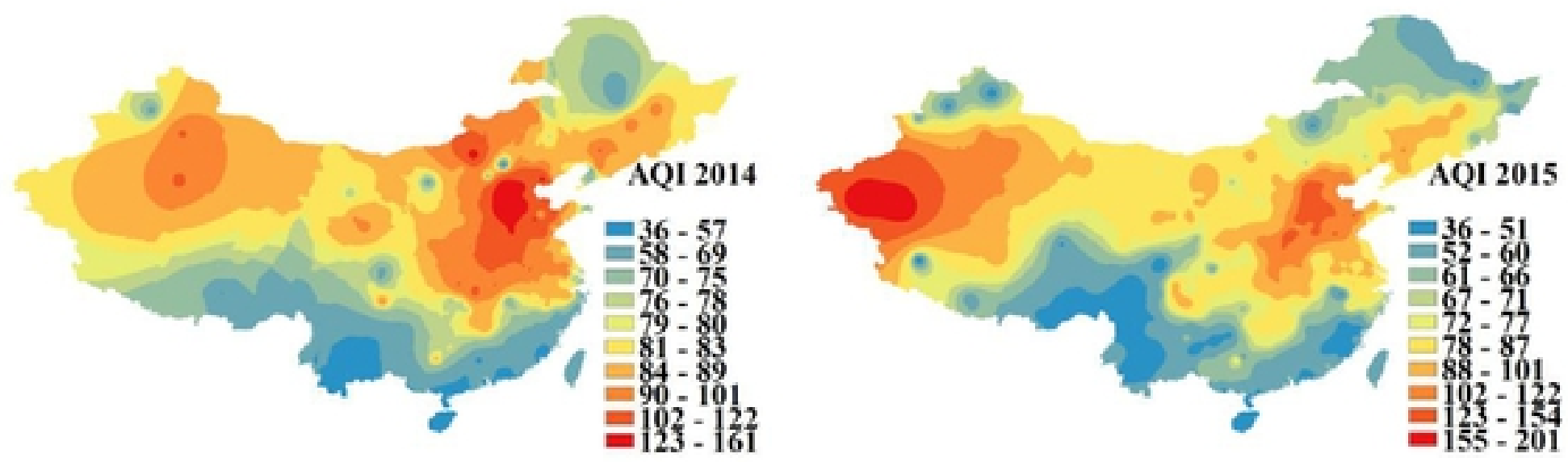

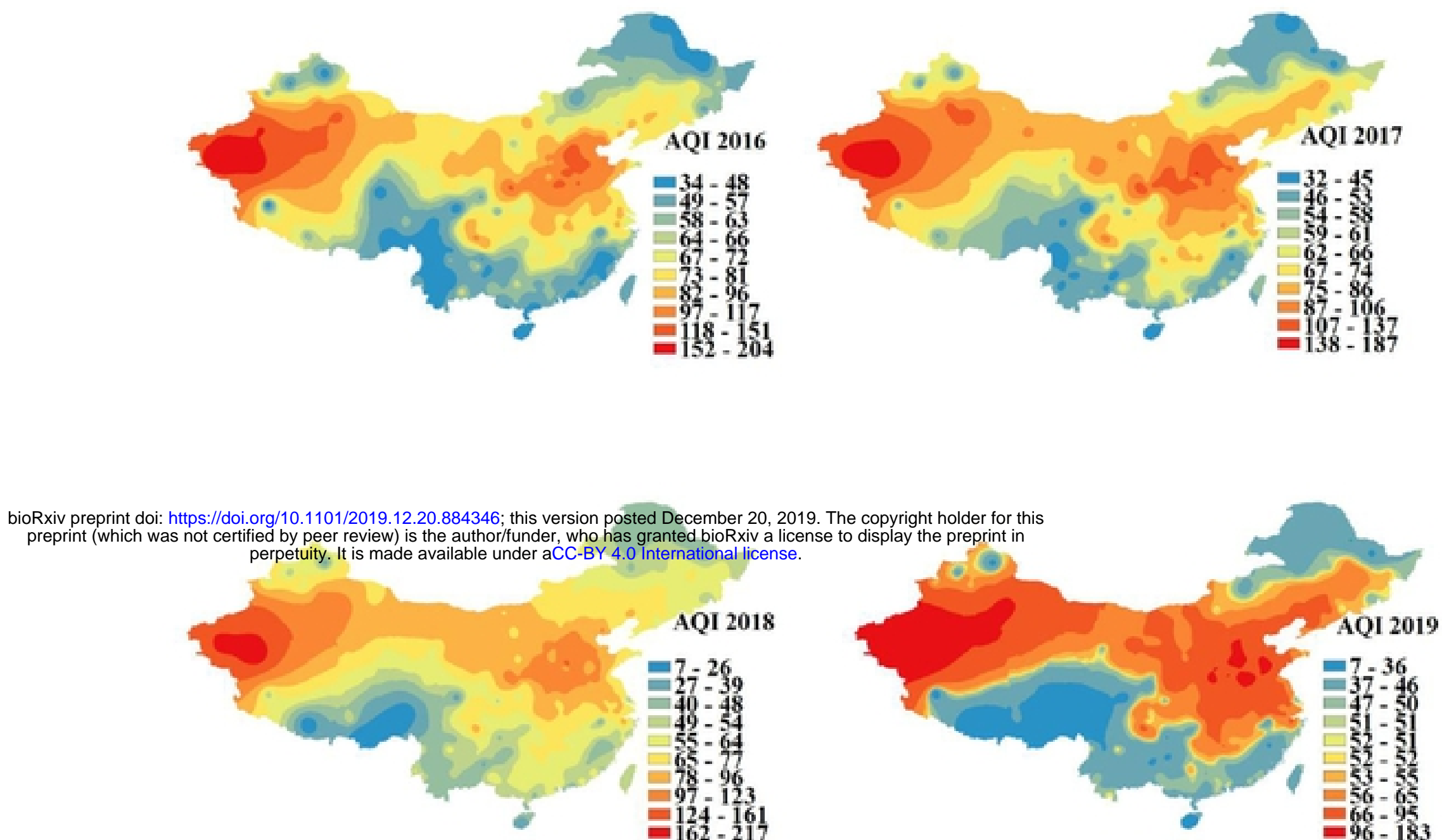

Figure 2. The spatial-temporal variation of the annual average AQI in mainland China from 2014 to 2019
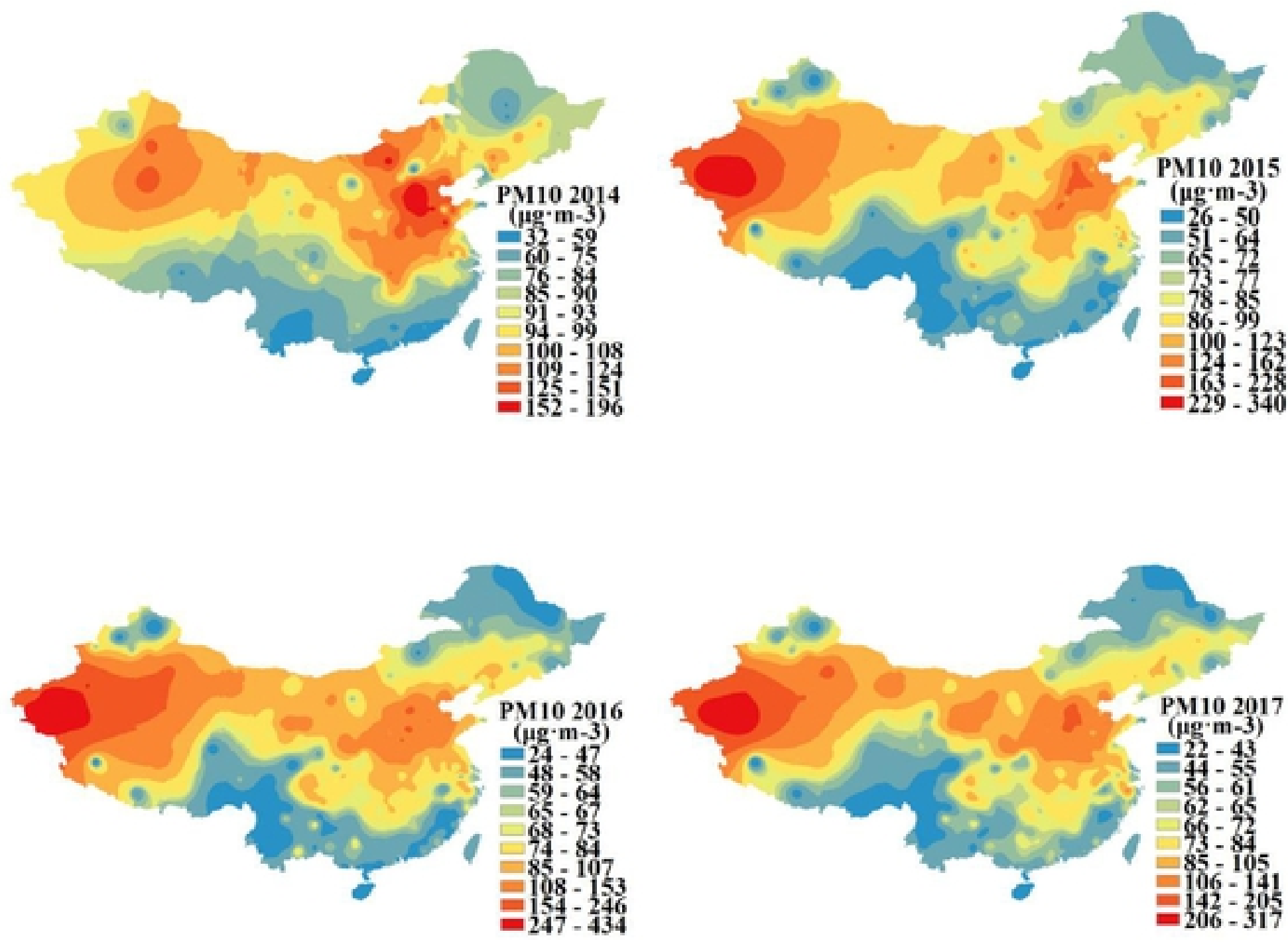

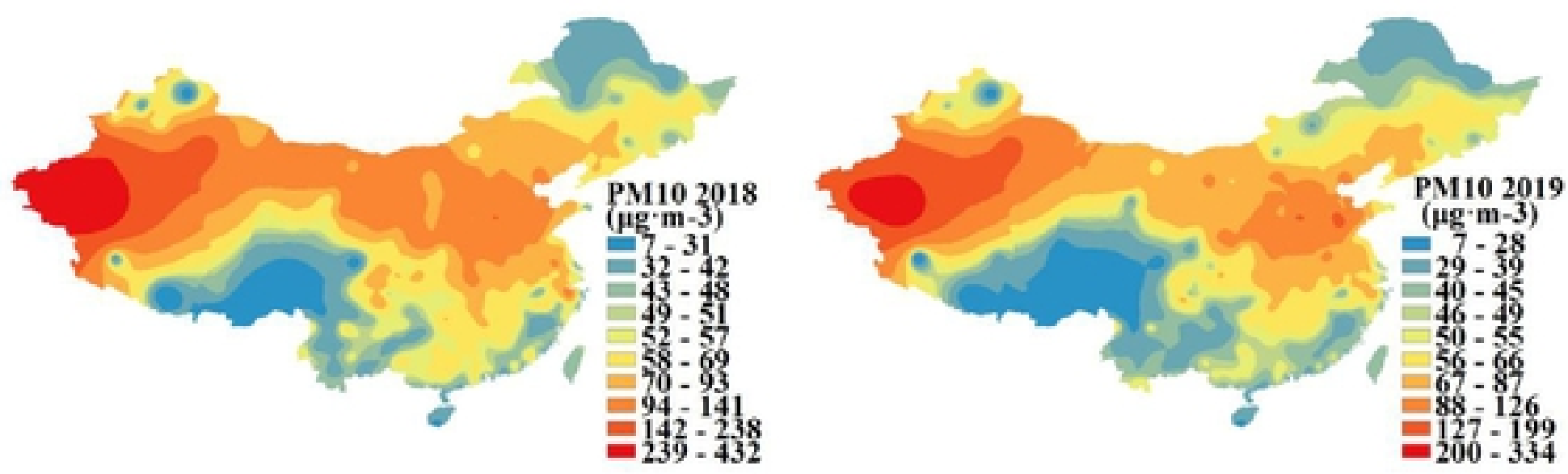

Figure 3. The spatial-temporal variation of the annual average mass

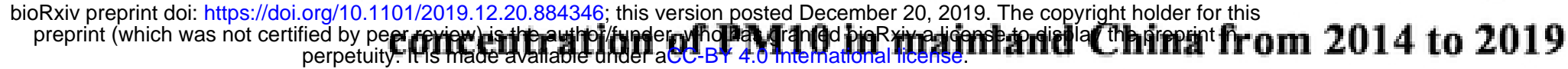
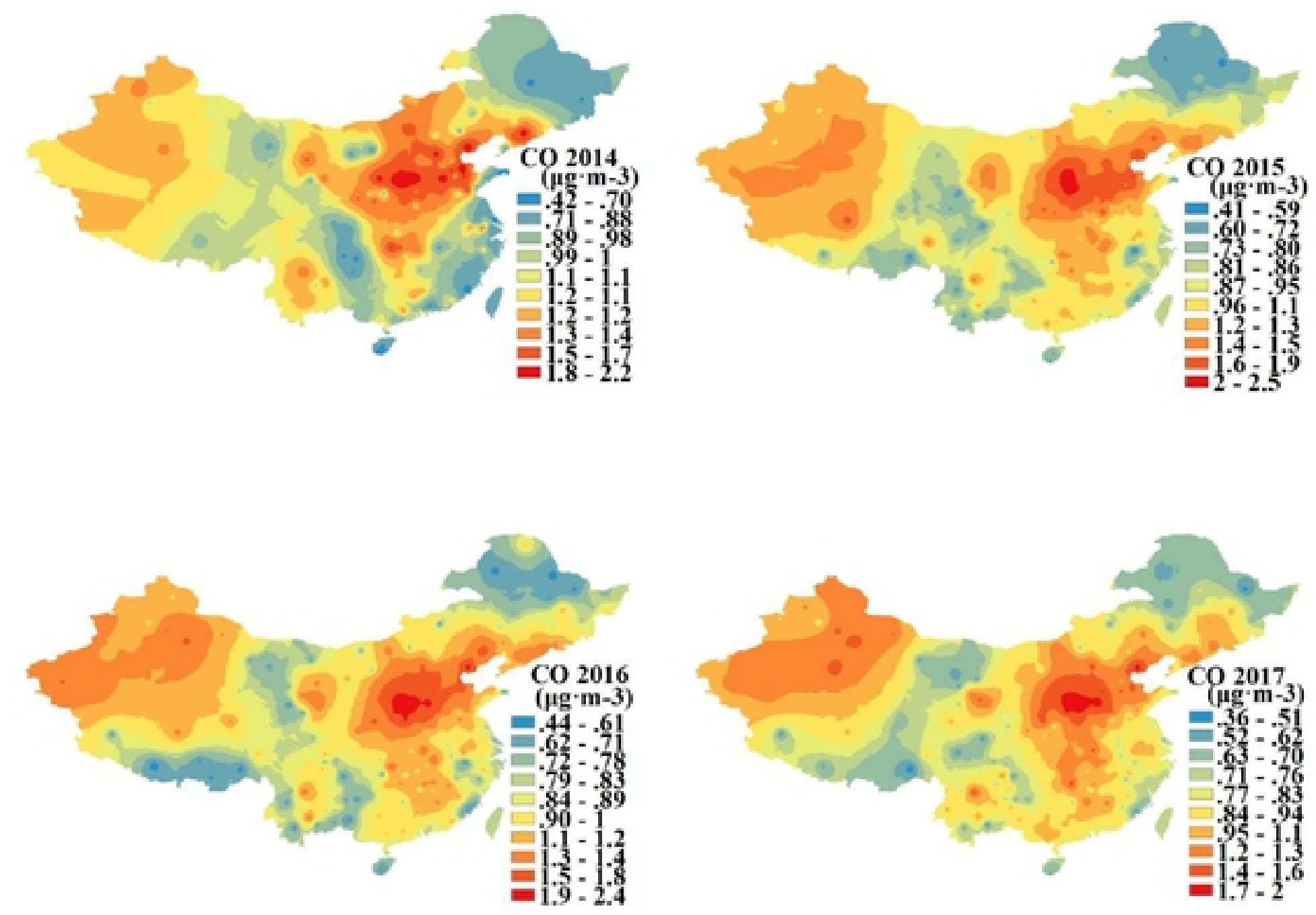

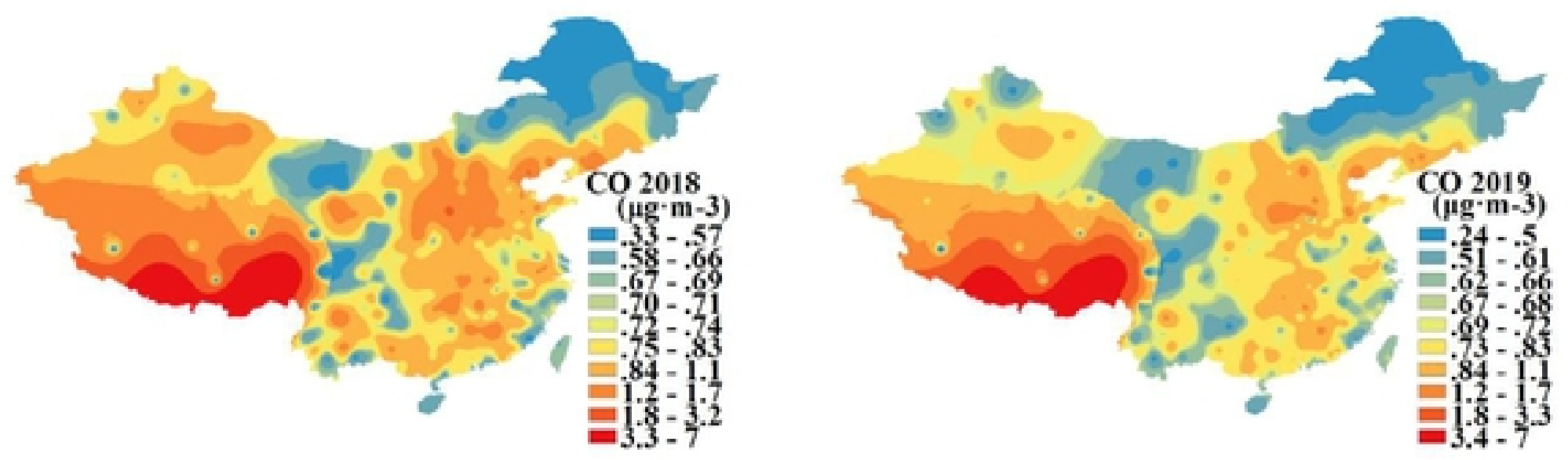

Figure 4. The spatial-temporal variation of the mass concentration of $\mathrm{CO}$ in

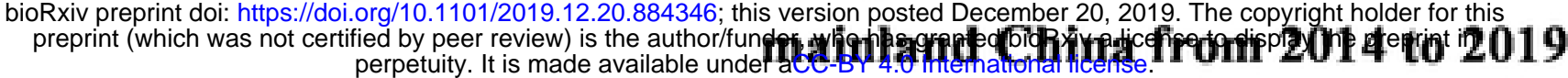
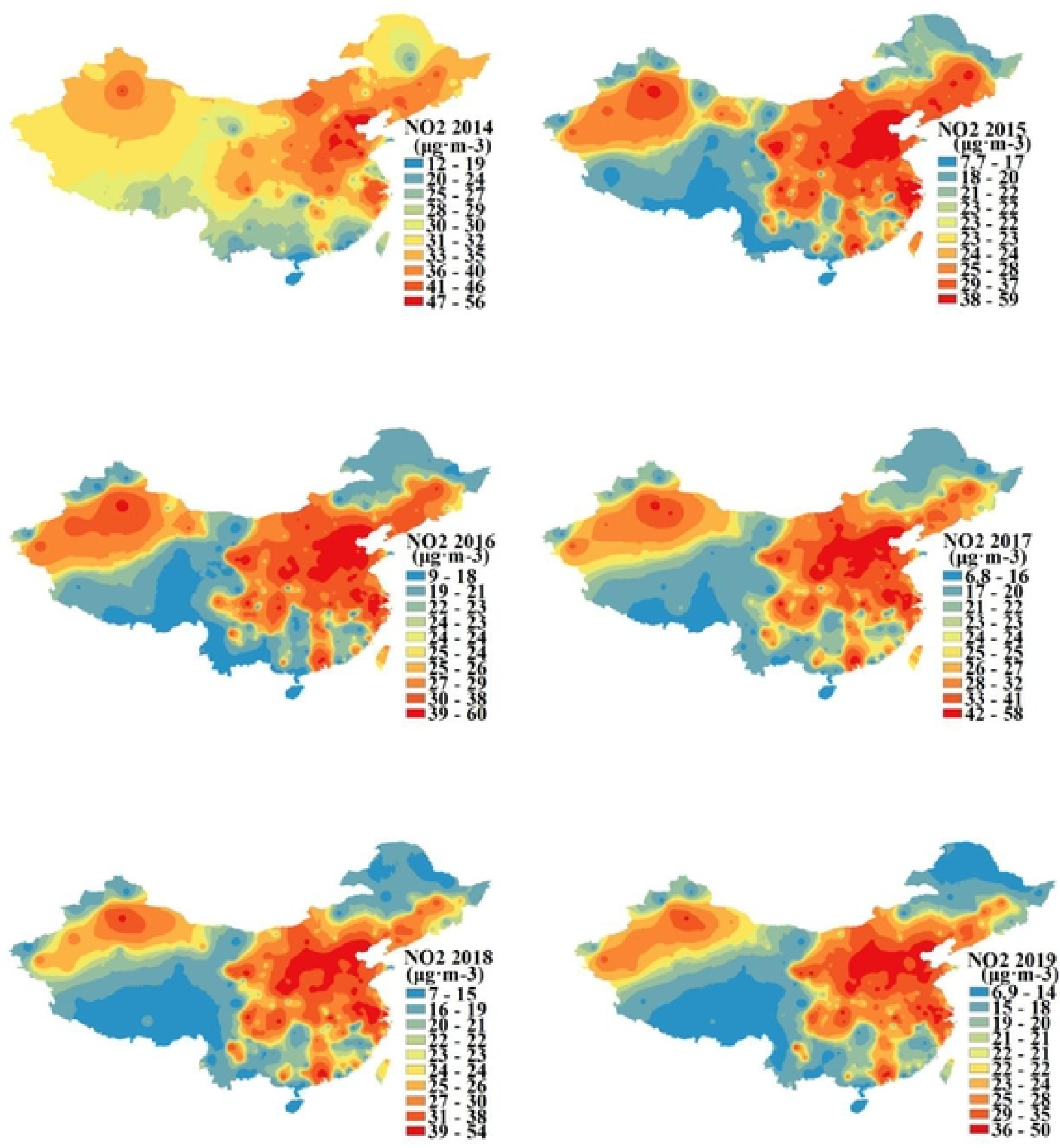
Figure 5. The spatial-temporal variation of the mass concentration of $\mathrm{NO2}$ in mainland China from 2014 to 2019

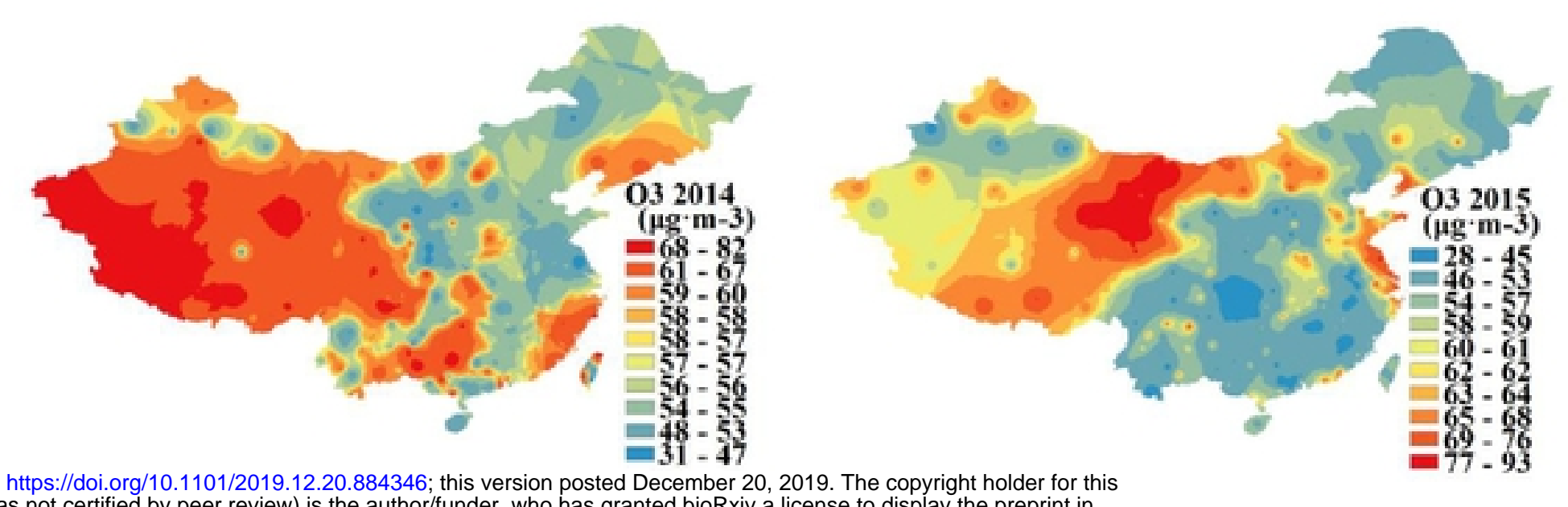

bioRxiv preprint doi: https://doi.org/10.1101/2019.12.20.884346; this version posted December 20, 2019. The copyright holder for this
preprint (which was not certified by per revis perpetuity. It is made available under aCC-BY 4.0 International license.
ing a licen
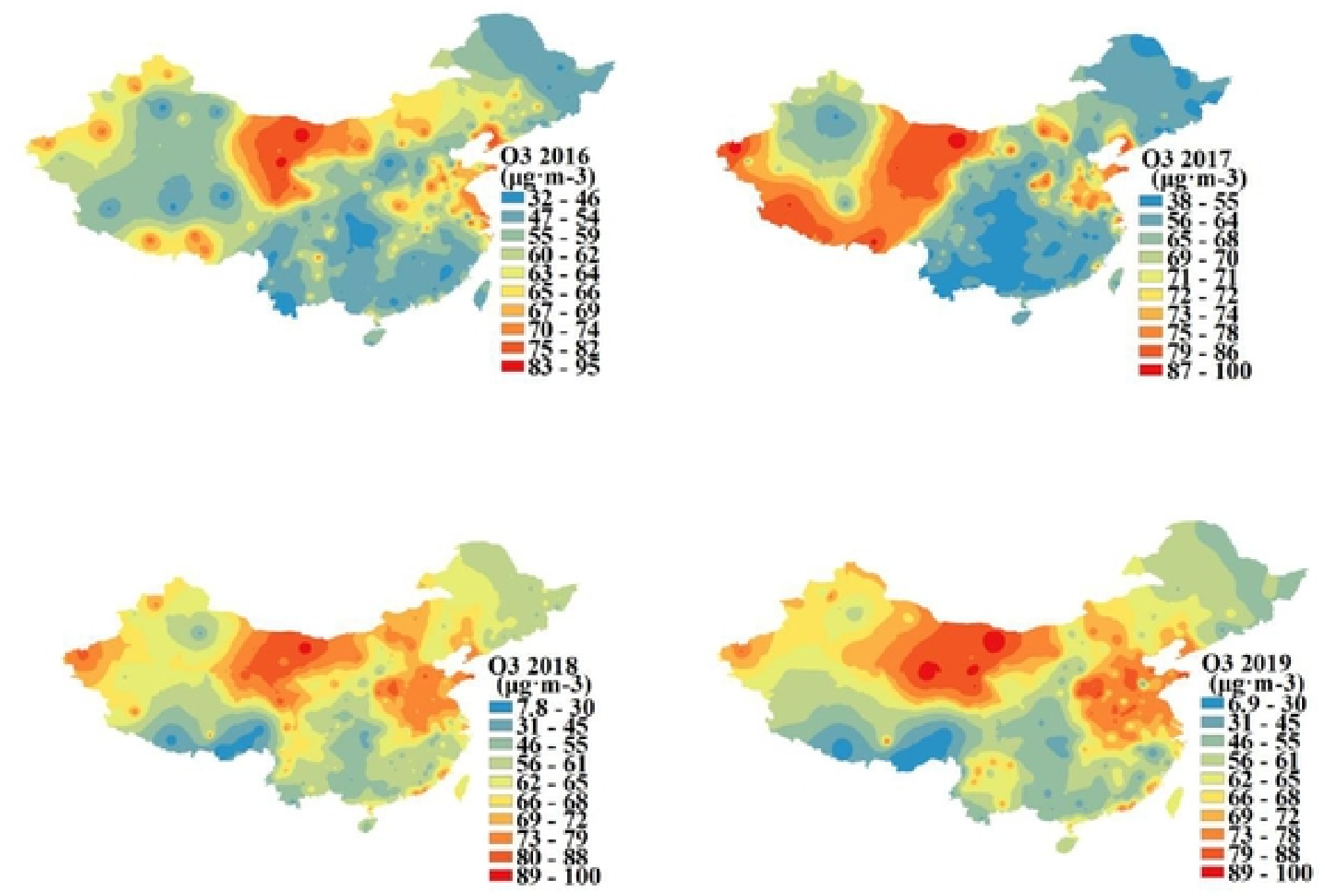

Figure 6. The spatial-temporal variation of the mass concentration of $\mathrm{O3}$ in mainland China from 2014 to 2019 

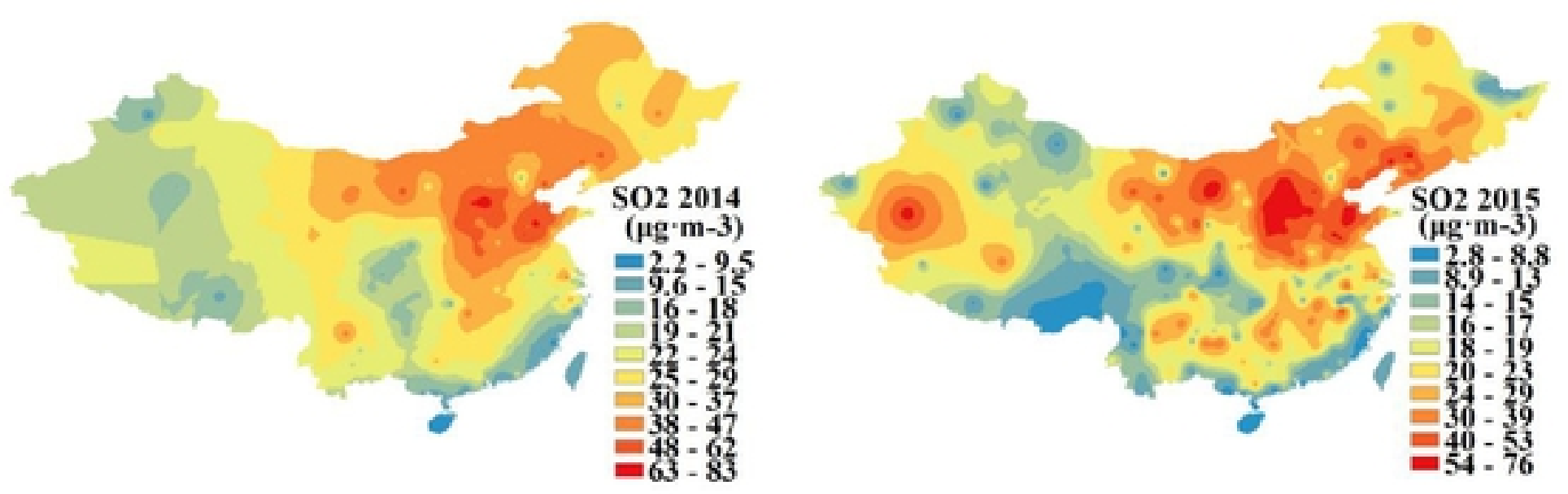
bioRxiv preprint doi: https://doi.org/10.1101/2019.12.20.884346; this version posted December 20, 2019. The copyright holder for this
preprint (which was not certified by peer review) is the author/funder, who has granted bioRxiv a license to display the preprint in
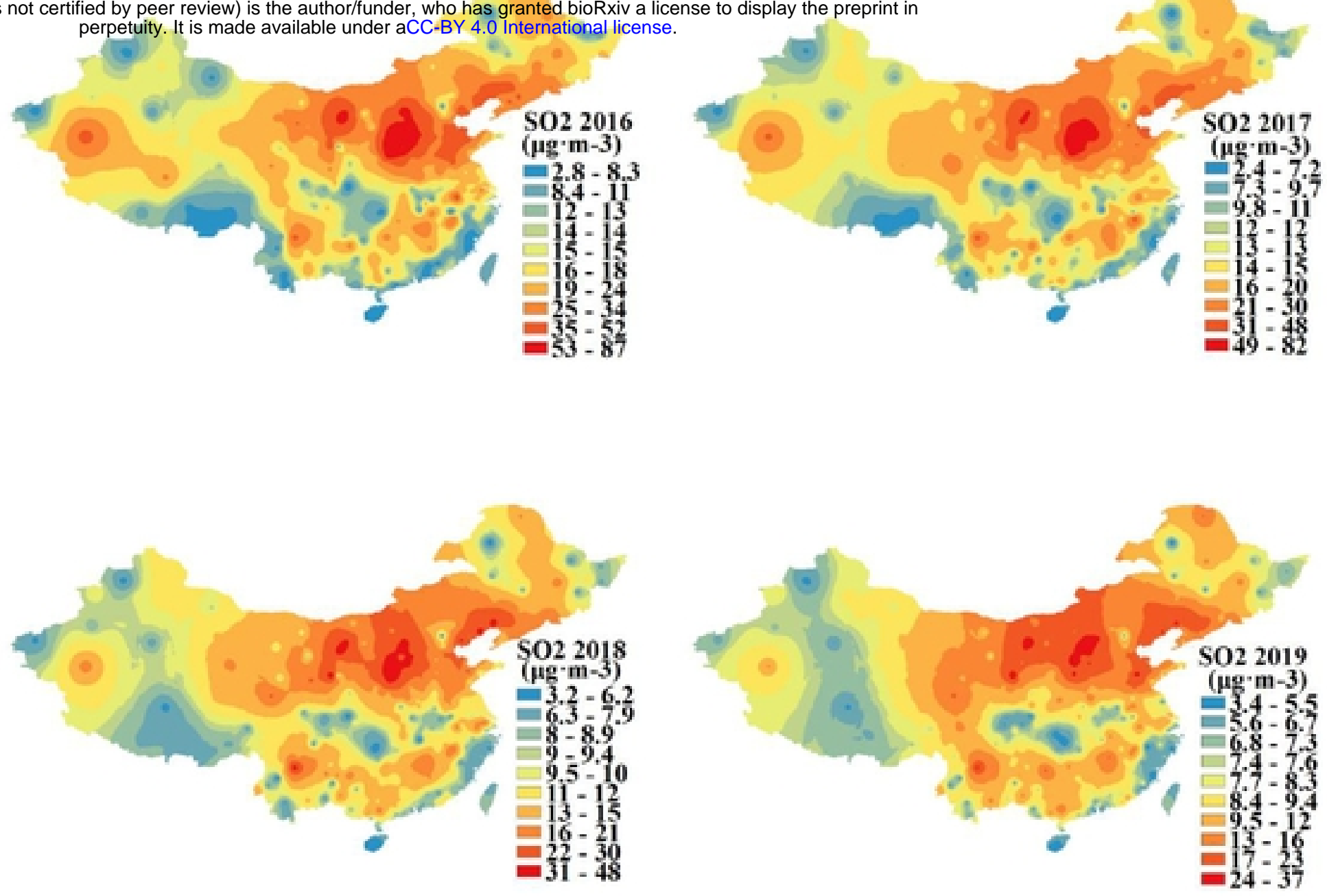